\title{
Dynamic Modeling and Stability Analysis of a Flexible Spinning Missile under Thrust
}

\author{
Tianfu Xu, Jili Rong", Dalin Xiang, Chenglong Pan, Xinzhe Yin \\ Department of Mechanics, Beijing Institute of Technology, No.5 Zhongguancun South Street, Haidian District, \\ Beijing 100081, China \\ *Corresponding author. Tel.: +86-10-68912732; Fax: +86-10-68914538, E-mail address: rongjili@ bit.edu.cn
}

\begin{abstract}
The general equations of motion of a flexible spinning missile under thrust in the powered flight phase are established and the stability of the motion of the missile is analyzed. The spinning missile is approximated to the unconstrained flexible rotor. Moreover, the thrust in the powered flight phase is deemed as a follower load when the factors of gyroscopic effect, aeroelastic effect, and axial force are considered under the mean axis condition. The equations of motion and stability of the flexible spinning missile in the powered flight phase are then deduced. The stability and dynamic response of the flexible spinning missile under thrust is analyzed through numerical calculation. Calculation results show that thrust, spinning speed, and dynamic pressure exert different influences on the stability of the spinning missile. These factors should be considered and analyzed comprehensively.
\end{abstract}

Keywords: follower thrust; flexible spinning missile; nonspinning coordinate system; stability; Timoshenko beam

\section{Nomenclature}

$\begin{array}{ll}A & =\text { cross section area } \\ a_{i}, b_{i} & =\text { generalized coordinates in } y, z \text { directions } \\ \{\boldsymbol{a}\},\{\boldsymbol{b}\} & =\text { generalized coordinate vectors in } y, z \text { directions } \\ {\left[\boldsymbol{C}_{D}\right]} & =\text { damping matrix } \\ d & =\text { diameter of missile } \\ F_{A y}, F_{A z} & =\text { lateral aerodynamic forces in } y, z \text { directions } \\ F_{D_{1}} & =\text { concentrated drag force at nose } \\ F_{G x}, F_{G y}, F_{G z} & =\text { components of gravity in } x, y, z \text { directions } \\ f_{A y}, f_{A z} & =\text { distributed lateral aerodynamic forces in } y, z \text { directions } \\ f_{D_{2}}(x, t) & =\text { distributed drag along longitudinal axis of missile } \\ \boldsymbol{i}, \boldsymbol{j}, \boldsymbol{k} & =\text { base vectors of nonspinning coordinate system } \\ \boldsymbol{i}_{0}, \boldsymbol{j}_{0}, \boldsymbol{k}_{0} & =\text { base vectors of Earth-fixed coordinate system } \\ \boldsymbol{i}^{\prime}, \boldsymbol{j}^{\prime}, \boldsymbol{k}^{\prime} & =\text { base vectors of thin disk coordinate system }\end{array}$

(C) 2016. This manuscript version is made available under the Elsevier user license http://www.elsevier.com/open-access/userlicense/1.0/ 


$$
\begin{aligned}
& \mathrm{i} \quad=\sqrt{-1} \\
& {\left[J_{p}\right] \quad=\text { gyroscopic matrix }} \\
& J_{x}, J_{y} \quad=\text { polar moment of inertia and diametric moment of inertia } \\
& j_{p}, j_{d} \quad=\text { per unit length polar moment of inertia and diametric moment of inertia } \\
& {[\boldsymbol{K}] \quad=\text { stiffness matrix }} \\
& {\left[\boldsymbol{K}_{D}\right]=\text { stiffness matrix caused by drag force at nose }} \\
& {\left[\boldsymbol{K}_{P}\right] \quad=\text { stiffness matrix caused by thrust }} \\
& {\left[\boldsymbol{K}_{P_{N}}\right] \quad=\text { stiffness matrix caused by axial force }} \\
& l_{b} \quad=\text { total length of missile } \\
& l_{\alpha}(x), l_{\beta}(x)=\text { lift and side force derivative per unit length } \\
& {[\boldsymbol{M}] \quad=\text { mass matrix }} \\
& \mathrm{Ma}=\text { Mach number } \\
& M_{A x}, M_{A y}, M_{A z}=\text { aerodynamic moments about x, y, z, respectively } \\
& M_{D y}, M_{D z}=\text { moments of drag around } y \text { and } z \text {, respectively } \\
& M_{P y}, M_{P z}=\text { moments of thrust around } y \text { and } z \text {, respectively } \\
& m_{b} \quad=\text { mass of missile } \\
& \boldsymbol{P} \quad=\text { thrust vector } \\
& P \quad=\text { magnitude of thrust } \\
& P_{N}(x, t) \quad=\text { distributed axial force } \\
& P_{x}, P_{y}, P_{z}=\text { components of thrust in } x, y, z \text { directions } \\
& \left\{\boldsymbol{Q}_{A y}\right\},\left\{\boldsymbol{Q}_{A z}\right\}=\text { transverse generalized forces in } y, z \text { directions } \\
& \{\boldsymbol{q}\}=\text { complex generalized coordinates, }\{\boldsymbol{a}\}+\mathrm{i}\{\boldsymbol{b}\} \\
& r_{0} \quad=\text { position vector of center of mass of spinning missiles } \\
& \boldsymbol{r}_{x} \quad=\text { longitudinal position vector of thin disk } \\
& \boldsymbol{u} \quad=\text { transverse elastic displacement vector of thin disk } \\
& v=\text { velocity vector of missile } \\
& \alpha, \beta=\quad \text { local angle of attack and angle of sideslip } \\
& \alpha_{0}, \beta_{0} \quad=\text { angle of attack and angle of sideslip of rigid missile } \\
& \delta=\text { complex angle of attack, } \beta_{0}+\mathrm{i} \alpha_{0} \\
& \vartheta, \psi, \varphi=\text { angle of pitch, angle of yaw, roll angle, respectively }
\end{aligned}
$$




$$
\begin{array}{ll}
\theta_{y}, \theta_{z} & =\text { elastic rotation angle of thin disk about } \mathrm{y}, \mathrm{z} \text {, respectively } \\
\lambda_{B} & =\text { fineness ratio } \\
\mu_{i} & =\text { critical damping ratio } \\
\rho & =\text { material density } \\
{[\boldsymbol{\Phi}],[\boldsymbol{\Psi}]} & =\text { displacement and rotation angle shape matrix } \\
\phi_{i}(x) & =\text { th displacement mode shape } \\
\Omega & =\text { spinning speed } \\
\omega_{0} & =\text { rotation angular velocity of nonspinning coordinate system relative to Earth-fixed coordinate } \\
\text { system } & =\text { rotation angular velocity of thin disk coordinate system relative to nonspinning coordinate system } \\
\boldsymbol{\omega}_{b} & =\text { complex rigid rotation angular velocity, } \omega_{0 y}+\mathrm{i} \omega_{0 z} \\
\omega_{0} & =\text { ith order natural frequency } \\
\omega_{i} & =\text { ith order forward and backward precession frequencies of elastic spinning missile, respectively } \\
\omega_{F i}, \omega_{B i} & \text { rigid forward and backward precession frequencies of rigid missile } \\
\omega_{R F}, \omega_{R B} &
\end{array}
$$

\section{Introduction}

Several modern spinning missiles apply large fineness ratio in their design. The elastic characteristics of missile structures are evident, and thus, researchers have paid attention to the resulting aeroelasticity of spinning missiles. Platus [1] derived the equations of motion and the aeroelastic stability equation of a flexible spinning missile using a simple beam model and analyzed the aeroelastic stability of slender spinning missiles. Mikhail $[2,3]$ studied the inflight flexure and spin lock-in of anti-tank kinetic energy projectiles. Murphy and Mermagen [4-6] discussed the flight motions of symmetric and finned elastic missiles as well as analyzed the resonance response and spin lock-in of a spinning missile under aerodynamic forces. Jegarkandi et al. [7] discussed aeroelastic stability of a flexible supersonic flight vehicle using nonlinear dynamics, nonlinear aerodynamics, and a linear structural model. Kitson and Cesnik [8] presented the development of the vehicle model and the computational framework to analyze the aeroelastic response of the maneuvering vehicle in free flight. Shi et al. [9] derived the nonlinear elastic dynamics equations of a supersonic and high fineness ratio spinning missile, and investigated the aeroelasticity affect the angular motions of a spinning missile. These studies have not considered the effect of the thrust in the powered flight phase.

Beal $[10,11]$ deduced the equations of motion of a flexible missile by considering thrust; he then analyzed the influence of the control system on the critical thrust value. In particular, this previous study identified whether thrust, as a follower load, would provide different conclusions when analyzing the vibration characteristics and dynamic stability of a structure [12]. The thrust stability of missiles has been widely research [13-15]; however, these studies have not considered the effects of spin and aeroelasticity. 
Pourtakdoust and Assadian [16] investigated the bending vibration characteristics of an elastic guided missile under thrust. However, the effect of spin was not discussed in the result analysis. Xie et al. $[17,18]$ considered aerodynamic force to derive the aeroelastic equation of a nonspinning and non-uniform beam under follower thrust. They discussed the transverse vibration, dynamic stability and flutter characteristics of a flexible missile with constant thrust. Trikha et al. $[19,20]$ used two methods to deduce the equations of motion of nonspinning slender aerospace vehicles and analyzed the effect of follower thrust on structure stability. Hodges [21] discussed several issues within one framework, in which the structural part is based on a mixed variational formulation which is geometrically-exact and based on finite elements and aerodynamic theories which vary according to the flight regime and missile geometry, and investigated the effects of follower forces on aeroelastic stability of flexible missiles. Rasti and Fazelzadeh [22] investigated multibody dynamic modeling and flutter analysis of a flexible slender vehicle based on the hybrid equations of motion in terms of quasi-coordinates. Oliveira et al. [23] presented the development of a three degree-of-freedom dynamic model of a sounding rocket that includes the elastic deformation parameters in the diff erential equations of motion solved during the trajectory computation.

Livshits et al. [24] studied the dynamic stability of free-flight rockets under thrust and roll rate as well as analyzed the dynamic response of imperfection factors in the time domain. Yoon and Kim [25] investigated the dynamic stability of a spinning unconstrained beam that was subjected to a pulsating follower force. However, they did not consider the effects of rigid motion and aeroelasticity.

On the basis of the aforementioned studies, this research considered the effects of spin, thrust, and aeroelasticity to approximate an elastic spinning missile to an unconstrained flexible rotor model. A nonspinning coordinate system was used under mean axis conditions. Furthermore, the general equations of motion and stability of a flexible spinning missile were deduced under follower thrust in the powered flight phase. The stability and dynamic response of the flexible spinning missile under thrust were analyzed through numerical calculations.

\section{Coordinate systems}

In this study, three kinds of coordinate systems are used to describe the general motion of flexible spinning missile: an Earth-fixed coordinate system $O_{0} x_{0} y_{0} z_{0}$, a nonspinning coordinate system $O x y z$, and a thin disk coordinate system $O^{\prime} \xi \eta \zeta$, as shown in Fig. 1.

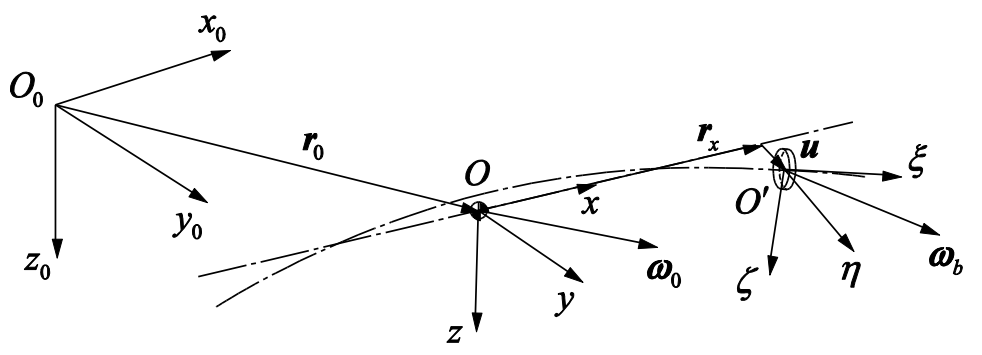

Fig. 1 Coordinate systems of flexible spinning missile.

The Earth-fixed coordinate system $O_{0} x_{0} y_{0} z_{0}$ is regarded as inertia coordinate system, and its base vectors are $\boldsymbol{i}_{0}, \boldsymbol{j}_{0}$, and $\boldsymbol{k}_{0}$. 
The origin $O$ of the nonspinning coordinate system is at instantaneous center of mass of missile; $O x$ coincides with mean axis of missile, and the positive direction is pointing to the front; $O z$ is in vertical plane of mean axis of missile and is perpendicular to the $O x$, and the positive direction is pointing to the down side; $O y$ is perpendicular to the other two axes and constitutes a right-handed coordinate system. The base vectors of this system are $i, j$, and $\boldsymbol{k}$. Based on the definition of the nonspinning coordinate system, the missile can rotate along with $O x$ of the nonspinning coordinate system. The nonspinning coordinate system $O x y z$ is under mean axis conditions; hence, the total angular momentum and the total kinetic energy of the system can both be simplified to reduce coupling between the rigidity and flexibility degree of freedom.

As shown in Fig. 2, the attitude angles of the spinning missile are determined by the pitching angle $\vartheta$, the yaw angle $\psi$, and the transformation matrix $\left[\boldsymbol{L}_{\vartheta \psi}\right]$ between an Earth-fixed coordinate system and a nonspinning coordinate system. Hence,

$$
\begin{gathered}
\left\{\begin{array}{l}
\boldsymbol{i} \\
\boldsymbol{j} \\
\boldsymbol{k}
\end{array}\right\}=\left[\boldsymbol{L}_{\vartheta \psi}\right]\left\{\begin{array}{l}
\boldsymbol{i}_{0} \\
\boldsymbol{j}_{0} \\
\boldsymbol{k}_{0}
\end{array}\right\}, \\
{\left[\boldsymbol{L}_{\vartheta \psi}\right]=\left[\begin{array}{ccc}
\cos \vartheta \cos \psi & \cos \vartheta \sin \psi & -\sin \vartheta \\
-\sin \psi & \cos \psi & 0 \\
\sin \vartheta \cos \psi & \sin \vartheta \sin \psi & \cos \vartheta
\end{array}\right] .}
\end{gathered}
$$

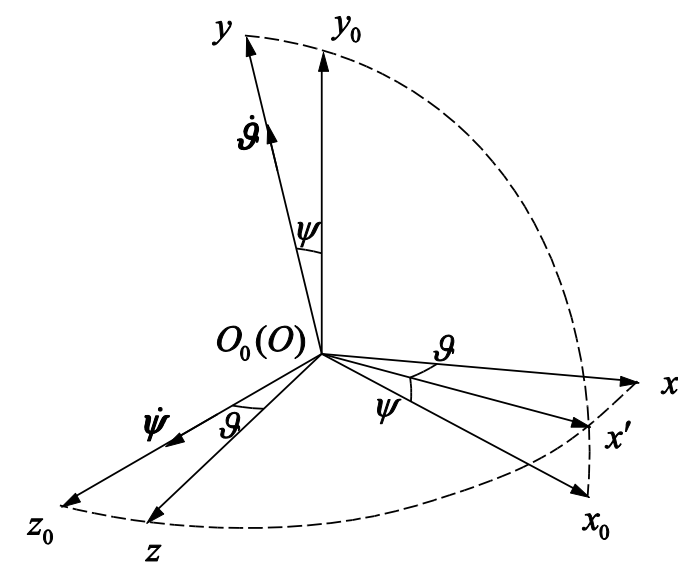

\section{Fig. 2 Transformation relationship between the Earth-fixed coordinate system and the nonspinning coordinate system.}

A thin disk thickness $\mathrm{dx}$ is sliced along the longitudinal direction of missile, as shown in Fig. 1. Coordinate system $O^{\prime} \xi \eta \zeta$ is fixed to the thin disk, and the origin of coordinate $O^{\prime}$ locates in center section of the thin disk; $O^{\prime} \xi$ is perpendicular to the cross section of the thin disk and is oriented along the missile longitudinal axis; $O^{\prime} \zeta$ is perpendicular to $O^{\prime} \xi$ in the cross section; $O^{\prime} \eta$ locates in the cross section and constitutes a right-handed coordinate system with the other two axes. The base vectors of the thin disk coordinate system are $\boldsymbol{i}^{\prime}, \boldsymbol{j}^{\prime}$, and $\boldsymbol{k}^{\prime}$. 
The transformation relationship between the nonspinning coordinate system and the thin disk coordinate system is shown in Fig. 3. Turning around the system of third axis, second axis, and first axis rotates $\theta_{z}, \theta_{\eta^{\prime}}$, and $\varphi$, respectively. The transformation matrix $\left[\boldsymbol{L}_{\varphi \theta_{\eta} \theta_{z}}\right]$ is between a nonspinning coordinate system and a thin disk coordinate system. Hence,

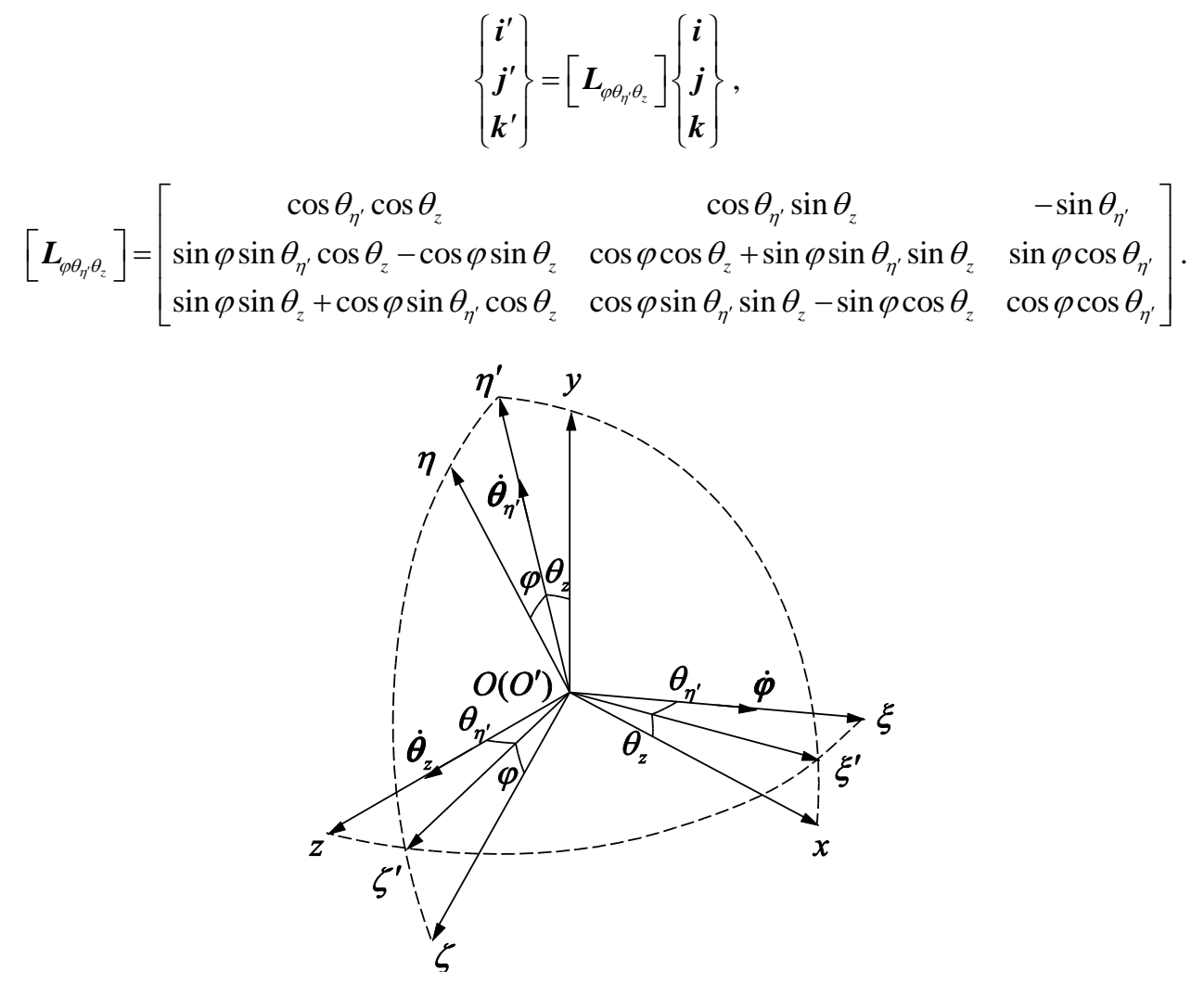

Fig. 3 Transformation relationship between the nonspinning coordinate system and the thin disk coordinate system.

\section{Equations of motion}

\subsection{Kinetic energy}

The total kinetic energy of the missile comprises the translational kinetic energy of center of mass and the rotational kinetic energy of around center of mass. It can be obtained by integrating the translational and rotational kinetic energy of the thin disk.

The translational kinetic energy of the thin disk is

$$
\mathrm{d} T_{T}=\frac{1}{2} \rho A v \cdot v \mathrm{~d} x,
$$

where $v$ is the absolute moving velocity of the thin disk center, $\rho$ is material density, and $A$ is cross section area. As shown in Fig. 1, the relationship between the position vectors is 


$$
\begin{gathered}
\boldsymbol{r}=\boldsymbol{r}_{0}+\boldsymbol{r}_{x}+\boldsymbol{u}, \\
\boldsymbol{v}=\frac{\mathrm{d} \boldsymbol{r}}{\mathrm{d} t}=\dot{\boldsymbol{r}}_{0}+\omega_{0} \times\left(\boldsymbol{r}_{x}+\boldsymbol{u}\right)+\dot{\boldsymbol{u}},
\end{gathered}
$$

where $\boldsymbol{r}_{0}$ is position vector of center of mass of spinning missiles in the Earth-fixed coordinate system, $\boldsymbol{r}_{x}$ is longitudinal position vector of the thin disk in the nonspinning coordinate system, and $\boldsymbol{u}$ is transverse elastic displacement vector of the thin disk in the nonspinning coordinate system. $\omega_{0}$ is rotation angular velocity of the nonspinning coordinate system relative to the Earth-fixed coordinate system, which denotes rigid rotation except for rotation along longitudinal axis of missile. The dot above the vector denotes relative derivative of vector for time in the respective coordinate system.

The total translational kinetic energy of the missile is obtained as

$$
T_{T}=\frac{1}{2} \int_{l_{b}} \rho A\left\{\boldsymbol{v}_{0} \cdot \boldsymbol{v}_{0}+\dot{\boldsymbol{u}} \cdot \dot{\boldsymbol{u}}+\left[\omega_{0} \times\left(\boldsymbol{r}_{x}+\boldsymbol{u}\right)\right] \cdot\left[\omega_{0} \times\left(\boldsymbol{r}_{x}+\boldsymbol{u}\right)\right]+2 \boldsymbol{v}_{0} \cdot \dot{\boldsymbol{u}}+2 \boldsymbol{\omega}_{0} \times\left(\boldsymbol{r}_{x}+\boldsymbol{u}\right) \cdot \boldsymbol{v}_{0}+2 \boldsymbol{\omega}_{0} \times\left(\boldsymbol{r}_{x}+\boldsymbol{u}\right) \cdot \dot{\boldsymbol{u}}\right\} \mathrm{d} x
$$

where $l_{b}$ is total length of the missile.

Mean axis needs to meet the condition that total translational momentum and total angular momentum in the coordinate system are always zero [26]. According to the mean axis conditions,

$$
\begin{gathered}
\int_{l_{b}} \rho A \boldsymbol{r}_{x} \mathrm{~d} x=\mathbf{0}, \int_{l_{b}} \rho A \boldsymbol{u} \mathrm{d} x=\mathbf{0}, \int_{l_{b}} \rho A \boldsymbol{r}_{x} \times \boldsymbol{u} \mathrm{d} x=\mathbf{0}, \\
\int_{l_{b}} \rho A \dot{\boldsymbol{u}} \mathrm{d} x=\boldsymbol{0} \\
\int_{l_{b}} \rho A\left(\boldsymbol{r}_{x}+\boldsymbol{u}\right) \times \dot{\boldsymbol{u}} \mathrm{d} x=\boldsymbol{0} .
\end{gathered}
$$

Vectors of Eq. (8) written component in the nonspinning coordinate system are

$$
\begin{gathered}
\boldsymbol{v}_{0}=\left[\begin{array}{lll}
\boldsymbol{i} & \boldsymbol{j} & \boldsymbol{k}
\end{array}\right]\left[\begin{array}{lll}
v_{x} & v_{y} & v_{z}
\end{array}\right]^{\mathrm{T}}, \\
\boldsymbol{u}=\left[\begin{array}{lll}
\boldsymbol{i} & \boldsymbol{j} & \boldsymbol{k}
\end{array}\right]\left[\begin{array}{lll}
0 & u_{y} & u_{z}
\end{array}\right]^{\mathrm{T}}, \\
\boldsymbol{r}_{x}=\left[\begin{array}{lll}
\boldsymbol{i} & \boldsymbol{j} & \boldsymbol{k}
\end{array}\right]\left[\begin{array}{lll}
x & 0 & 0
\end{array}\right]^{\mathrm{T}}, \\
\boldsymbol{\omega}_{0}=\left[\begin{array}{lll}
\boldsymbol{i} & \boldsymbol{j} & \boldsymbol{k}
\end{array}\right]\left[\begin{array}{lll}
\omega_{0 x} & \omega_{0 y} & \omega_{0 z}
\end{array}\right]^{\mathrm{T}} .
\end{gathered}
$$

Obtained total translational energy of the missile is

$$
T_{T}=\frac{1}{2} m_{b}\left(v_{x}^{2}+v_{y}^{2}+v_{z}^{2}\right)+\frac{1}{2} \int_{l_{b}} \rho A\left[\left(\dot{u}_{y}^{2}+\dot{u}_{z}^{2}\right)+x^{2}\left(\omega_{0 y}^{2}+\omega_{0 z}^{2}\right)+\omega_{0 x}^{2}\left(u_{y}^{2}+u_{z}^{2}\right)+\left(\omega_{0 y} u_{z}-\omega_{0 z} u_{y}\right)^{2}\right] \mathrm{d} x
$$

Rotational kinetic energy of the thin disk is

$$
\mathrm{d} T_{R}=\frac{1}{2} \omega \cdot \mathrm{d} j_{b} \cdot \omega=\frac{1}{2}\left(\omega_{0}+\omega_{b}\right) \cdot \mathrm{d} j_{b} \cdot\left(\omega_{0}+\omega_{b}\right),
$$

where $\omega$ is the absolute angular velocity of the thin disk, $\omega=\omega_{0}+\omega_{b} ; \omega_{b}$ is rotation angular velocity of the thin disk coordinate system relative to the nonspinning coordinate system, which is composed of elastic rotation $\omega_{e}$ of 
the thin disk caused by elastic displacement of missile and spinning angular velocity $\boldsymbol{\Omega}$ along longitudinal axis of missile, $\boldsymbol{\omega}_{b}=\boldsymbol{\omega}_{e}+\boldsymbol{\Omega} . \mathrm{d}_{b}$ is inertia tensor of the thin disk. The thin disk coordinate system is principal axis system of the thin disk; hence, the matrix in the thin disk coordinate system of $\mathrm{d}_{b}$ can be denoted as

$$
\left[\mathrm{dj}_{b}\right]=\left[\begin{array}{lll}
j_{p} & & \\
& j_{d} & \\
& & j_{d}
\end{array}\right] \mathrm{d} x,
$$

where $j_{p}$ and $j_{d}$ are per unit length polar moment of inertia of spinning missiles and diametric moment of inertia of spinning missiles, respectively, $j_{p}=2 j_{d}=2 \rho I ; I$ is moment of inertia of cross section of the thin disk.

Components of $\omega_{0}$ and $\omega_{b}$ in the nonspinning coordinate system and in the thin disk coordinate system, respectively, are presented.

$$
\begin{aligned}
& \left\{\begin{array}{l}
\omega_{0 x} \\
\omega_{0 y} \\
\omega_{0 z}
\end{array}\right\}=\left\{\begin{array}{c}
-\dot{\psi} \sin \vartheta \\
\dot{\vartheta} \\
\dot{\psi} \cos \vartheta
\end{array}\right\} \\
& \left\{\begin{array}{l}
\omega_{0 \xi} \\
\omega_{0 \eta} \\
\omega_{0 \varsigma}
\end{array}\right\}=\left[\boldsymbol{L}_{\varphi \theta_{\eta} \theta_{z}}\right]\left[\begin{array}{l}
\omega_{0 x} \\
\omega_{0 y} \\
\omega_{0 z}
\end{array}\right\}, \\
& \left\{\begin{array}{c}
\omega_{b \xi} \\
\omega_{b \eta} \\
\omega_{b \zeta}
\end{array}\right\}=\left[\begin{array}{ccc}
1 & 0 & -\sin \theta_{\eta^{\prime}} \\
0 & \cos \varphi & \sin \varphi \cos \theta_{\eta^{\prime}} \\
0 & -\sin \varphi & \cos \varphi \cos \theta_{\eta^{\prime}}
\end{array}\right]\left\{\begin{array}{c}
\dot{\varphi} \\
\dot{\theta}_{\eta^{\prime}} \\
\dot{\theta}_{z}
\end{array}\right\}, \\
& \left\{\begin{array}{l}
\omega_{b x} \\
\omega_{b y} \\
\omega_{b z}
\end{array}\right\}=\left[\boldsymbol{L}_{\varphi \theta_{\eta^{\prime}} \theta_{z}}\right]^{\mathrm{T}}\left\{\begin{array}{l}
\omega_{b \xi} \\
\omega_{b \eta} \\
\omega_{b \zeta}
\end{array}\right\}=\left[\begin{array}{ccc}
\cos \theta_{\eta^{\prime}} \cos \theta_{z} & -\sin \theta_{z} & 0 \\
\cos \theta_{\eta^{\prime}} \sin \theta_{z} & \cos \theta_{z} & 0 \\
-\sin \theta_{\eta^{\prime}} & 0 & 1
\end{array}\right]\left\{\begin{array}{l}
\Omega \\
\dot{\theta}_{\eta^{\prime}} \\
\dot{\theta}_{z}
\end{array}\right\},
\end{aligned}
$$

where $\varphi$ is roll angle around longitudinal axis of the thin disk; $\theta_{z}$ is angle between $O x$ and projection of outside normal vector of the thin disk on $O x y$ plan of the nonspinning coordinate system, which denotes the elastic rotation of the thin disk around $O z$ axis; $\theta_{\eta^{\prime}}$ is rotation angle around $O^{\prime} \eta^{\prime}$ axis, and can be approximately taken when missile produces small deformation $\theta_{\eta^{\prime}} \approx \theta_{y} ; \theta_{y}$ is angle between $O x$ and projection of outside normal vector of the thin disk on $O x z$ plan of the nonspinning coordinate system, which denotes the elastic rotation of the thin disk around $O y$ axis. The relationship between spinning speed and roll angle of missile is

$$
\boldsymbol{\Omega}=\dot{\boldsymbol{\varphi}}, \Omega=\dot{\varphi} .
$$

Assuming the elastic deformation of missile is smaller, considering $\sin \theta_{\eta^{\prime}} \approx \theta_{\eta^{\prime}} \approx \theta_{y}, \sin \theta_{z} \approx \theta_{z}$, $\cos \theta_{\eta^{\prime}} \approx \cos \theta_{z} \approx 1$, obtained total rotational kinetic energy of missile is 


$$
\begin{aligned}
T_{R}= & \frac{1}{2} \int_{l_{b}} \rho I\left[\left(2 \Omega^{2}-4 \Omega \dot{\theta}_{z} \theta_{y}+\dot{\theta}_{y}^{2}+\dot{\theta}_{z}^{2}\right)+\left(2 \omega_{0 x}^{2}+\omega_{0 y}^{2}+\omega_{0 z}^{2}\right)+\left(\omega_{0 x}^{2}+2 \omega_{0 z}^{2}\right) \theta_{y}^{2}+\left(\omega_{0 x}^{2}+2 \omega_{0 y}^{2}\right) \theta_{z}^{2}+2 \omega_{0 x} \omega_{0 y} \theta_{z}\right. \\
& \left.-2 \omega_{0 x} \omega_{0 z} \theta_{y}-2 \omega_{0 y} \omega_{0 z} \theta_{y} \theta_{z}+4 \Omega\left(\omega_{0 x}+\omega_{0 y} \theta_{z}-\omega_{0 z} \theta_{y}\right)-2 \omega_{0 x}\left(\dot{\theta}_{z} \theta_{y}+\dot{\theta}_{y} \theta_{z}\right)+2 \omega_{0 y} \dot{\theta}_{y}+2 \omega_{0 z} \dot{\theta}_{z}\right] \mathrm{d} x .
\end{aligned}
$$

Elastic deformations of missile are expanded in sum of product of mode functions and generalized coordinates.

$$
u_{y}=\sum_{i=1}^{m} \phi_{i}(x) a_{i}(t)=[\boldsymbol{\Phi}]\{\boldsymbol{a}\}, u_{z}=\sum_{i=1}^{m} \phi_{i}(x) b_{i}(t)=[\boldsymbol{\Phi}]\{\boldsymbol{b}\},
$$

where $\phi_{i}(x)$ is the $i$ th displacement mode shape and $[\boldsymbol{\Phi}]$ is displacement shape matrix. According to the assumption that the missile has axisymmetric structures, the same shape function of $y$ and $z$ directions is used. $a_{i}(t)$ and $b_{i}(t)$ are corresponding generalized coordinates, and $\{\boldsymbol{a}\}$ and $\{\boldsymbol{b}\}$ are generalized coordinate vectors.

For Timoshenko beam model, rotation angle can be represented as

$$
\theta_{z}=[\boldsymbol{\Psi}]\{\boldsymbol{a}\}=\left(\left[\boldsymbol{\Phi}^{\prime}\right]+\frac{E I}{\kappa G A}\left[\boldsymbol{\Phi}^{\prime \prime \prime}\right]\right)\{\boldsymbol{a}\},-\theta_{y}=[\boldsymbol{\Psi}]\{\boldsymbol{b}\},
$$

where $[\boldsymbol{\Psi}]$ is rotation angle shape matrix. For Rayleigh beam model without considering shear deformation, is obtained $[\boldsymbol{\Psi}]=\left[\boldsymbol{\Phi}^{\prime}\right]$.

The kinetic energy can be represented as

$$
\begin{aligned}
& T=T_{T}+T_{R}=\frac{1}{2} m_{b}\left(v_{x}^{2}+v_{y}^{2}+v_{z}^{2}\right)+\frac{1}{2} J_{x}\left(\omega_{0 x}+\Omega\right)^{2}+\frac{1}{4}\left(J_{x}+2 J_{y}\right)\left(\omega_{0 y}^{2}+\omega_{0 z}^{2}\right) \\
&+\frac{1}{2}\{\dot{\boldsymbol{a}}\}^{\mathrm{T}}[\boldsymbol{M}]\{\dot{\boldsymbol{a}}\}+\frac{1}{2}\{\dot{\boldsymbol{b}}\}^{\mathrm{T}}[\boldsymbol{M}]\{\dot{\boldsymbol{b}}\}+\{\dot{\boldsymbol{a}}\}^{\mathrm{T}}\left(\Omega\left[\boldsymbol{J}_{p}\right]+\omega_{0 x}\left[\boldsymbol{M}_{R}\right]\right)\{\boldsymbol{b}\} \\
&+\omega_{0 x}\{\boldsymbol{a}\}^{\mathrm{T}}\left[\boldsymbol{M}_{R}\right]\{\dot{\boldsymbol{b}}\}+\frac{1}{2}\{\boldsymbol{a}\}^{\mathrm{T}}\left(\omega_{0 x}^{2}[\boldsymbol{M}]+\omega_{0 z}^{2}\left[\boldsymbol{M}_{T}\right]+\omega_{0 y}^{2}\left[\boldsymbol{J}_{p}\right]\right)\{\boldsymbol{a}\} \\
&+\frac{1}{2}\{\boldsymbol{b}\}^{\mathrm{T}}\left(\omega_{0 x}^{2}[\boldsymbol{M}]+\omega_{0 y}^{2}\left[\boldsymbol{M}_{T}\right]+\omega_{0 z}^{2}\left[\boldsymbol{J}_{p}\right]\right)\{\boldsymbol{b}\}+\omega_{0 y} \omega_{0 z}\{\boldsymbol{a}\}^{\mathrm{T}}\left(\left[\boldsymbol{M}_{R}\right]-\left[\boldsymbol{M}_{T}\right]\right)\{\boldsymbol{b}\} \\
&+\omega_{0 z}\left[\boldsymbol{I}_{0}\right]\{\dot{\boldsymbol{a}}\}-\omega_{0 y}\left[\boldsymbol{I}_{0}\right]\{\dot{\boldsymbol{b}}\}+\left(\omega_{0 x}+2 \Omega\right)\left(\omega_{0 y}\left[\boldsymbol{I}_{0}\right]\{\boldsymbol{a}\}+\omega_{0 z}\left[\boldsymbol{I}_{0}\right]\{\boldsymbol{b}\}\right),
\end{aligned}
$$

where

$$
\begin{gathered}
{[\boldsymbol{M}]=\left[\boldsymbol{M}_{T}\right]+\left[\boldsymbol{M}_{R}\right],\left[\boldsymbol{M}_{T}\right]=\int_{l_{b}} \rho A[\boldsymbol{\Phi}]^{\mathrm{T}}[\boldsymbol{\Phi}] \mathrm{d} x,} \\
{\left[\boldsymbol{M}_{R}\right]=\int_{l_{b}} \rho I[\boldsymbol{\Psi}]^{\mathrm{T}}[\boldsymbol{\Psi}] \mathrm{d} x,\left[\boldsymbol{J}_{p}\right]=2\left[\boldsymbol{M}_{R}\right],} \\
{\left[\boldsymbol{I}_{0}\right]=\int_{l_{b}} \rho I[\boldsymbol{\Psi}] \mathrm{d} x, J_{x}=\int_{l_{b}} 2 \rho I \mathrm{~d} x, J_{y}=\int_{l_{b}} \rho A x^{2} \mathrm{~d} x}
\end{gathered}
$$

\subsection{Elastic potential energy}


Transverse bending and shear deformation in the motion of missile must be considered to simplify the missile to Timoshenko beam model. Regardless of the longitudinal axial and torsion deformation, the elastic potential energy $U$ of missile can be expressed as

$$
U=\frac{1}{2} \int_{l_{b}}\left[E I \theta_{y}^{\prime 2}+E I \theta_{z}^{\prime 2}+\kappa G A\left(\theta_{y}+u_{z}^{\prime}\right)^{2}+\kappa G A\left(\theta_{z}-u_{y}^{\prime}\right)^{2}\right] \mathrm{d} x=\frac{1}{2}\{\boldsymbol{a}\}^{\mathrm{T}}[\boldsymbol{K}]\{\boldsymbol{a}\}+\frac{1}{2}\{\boldsymbol{b}\}^{\mathrm{T}}[\boldsymbol{K}]\{\boldsymbol{b}\},
$$

where

$$
[\boldsymbol{K}]=\int_{l_{b}}\left(E I\left[\boldsymbol{\Psi}^{\prime}\right]^{\mathrm{T}}\left[\boldsymbol{\Psi}^{\prime}\right]+\frac{E^{2} I^{2}}{\kappa G A}\left[\boldsymbol{\Phi}^{\prime \prime \prime}\right]^{\mathrm{T}}\left[\boldsymbol{\Phi}^{\prime \prime \prime}\right]\right) \mathrm{d} x
$$

\subsection{Damping energy}

Damping energy $D$ of missile structure is given by

$$
D=\frac{1}{2} \int_{l_{b}} c_{1}\left(\dot{u}_{y}^{2}+\dot{u}_{z}^{2}\right) \mathrm{d} x+\frac{1}{2} \int_{l_{b}} c_{2}\left(\dot{\theta}_{y}^{2}+\dot{\theta}_{z}^{2}\right) \mathrm{d} x=\frac{1}{2}\{\dot{\boldsymbol{a}}\}^{\mathrm{T}}[\boldsymbol{C}]\{\dot{\boldsymbol{a}}\}+\frac{1}{2}\{\dot{\boldsymbol{b}}\}^{\mathrm{T}}[\boldsymbol{C}]\{\dot{\boldsymbol{b}}\}
$$

where

$$
\left[\boldsymbol{C}_{D}\right]=\left[\boldsymbol{C}_{D 1}\right]+\left[\boldsymbol{C}_{D 2}\right]=\int_{l_{b}} c_{1}[\boldsymbol{\Phi}]^{\mathrm{T}}[\boldsymbol{\Phi}] \mathrm{d} x+\int_{l_{b}} c_{2}[\boldsymbol{\Psi}]^{\mathrm{T}}[\boldsymbol{\Psi}] \mathrm{d} x
$$

where $c_{1}$ and $c_{2}$ are damping coefficients. If the proportional damping is used, then the damping matrix $\left[\boldsymbol{C}_{D}\right]$ can be expressed as

$$
\left[\boldsymbol{C}_{D}\right]=\operatorname{diag}\left(2 \mu_{1} \omega_{1} M_{11}, 2 \mu_{2} \omega_{2} M_{22}, \cdots, 2 \mu_{i} \omega_{i} M_{i i} \cdots, 2 \mu_{m} \omega_{m} M_{m m}\right),
$$

where $\omega_{i}$ is the $i$ th natural frequency, $\mu_{i}$ is critical damping ratio of corresponding mode, and $M_{i i}$ is diagonal element of mass matrix $[\boldsymbol{M}]$.

\subsection{Works of thrust, drag, and axial force}

Thrust $\boldsymbol{P}$ is regarded as a steady follower load. Given that the deformation of the missile is small, the linearization components of thrust is obtained by

$$
\begin{aligned}
& \boldsymbol{P}=\left[\begin{array}{lll}
\boldsymbol{i} & \boldsymbol{j} & \boldsymbol{k}
\end{array}\right]\left[\begin{array}{lll}
P_{x} & P_{y} & P_{z}
\end{array}\right]^{\mathrm{T}} \\
& =\left[\begin{array}{lll}
\boldsymbol{i} & \boldsymbol{j} & \boldsymbol{k}
\end{array}\right]\left[\begin{array}{lll}
P & P \theta_{z}\left(x_{P}\right) & -P \theta_{y}\left(x_{P}\right)
\end{array}\right]^{\mathrm{T}}
\end{aligned}
$$

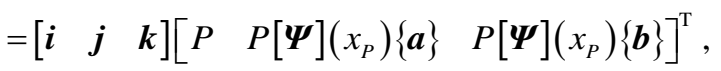

where $x_{P}$ is the axial position of the thrust application point.

The components of thrust moment $\boldsymbol{M}_{P}$ at the center of mass of the missile are

$$
\begin{gathered}
M_{P y}=P_{x} u_{z}\left(x_{P}, t\right)-P_{z} x_{P}=P\left[\boldsymbol{\Phi}\left(x_{P}\right)\right]\{\boldsymbol{b}\}-P x_{P}\left[\boldsymbol{\Psi}\left(x_{P}\right)\right]\{\boldsymbol{b}\}, \\
M_{P z}=-P_{x} u_{y}\left(x_{P}, t\right)+P_{y} x_{P}=-P\left[\boldsymbol{\Phi}\left(x_{P}\right)\right]\{\boldsymbol{a}\}+P x_{P}\left[\boldsymbol{\Psi}\left(x_{P}\right)\right]\{\boldsymbol{a}\} .
\end{gathered}
$$


The virtual work of the thrust is

$$
\delta W_{P}=P \theta_{z}\left(x_{P}, t\right) \delta u_{y}\left(x_{P}, t\right)-P \theta_{y}\left(x_{P}, t\right) \delta u_{z}\left(x_{P}, t\right)=\{\delta \boldsymbol{a}\}^{\mathrm{T}}\left[\boldsymbol{K}_{P}\right]\{\boldsymbol{a}\}+\{\delta \boldsymbol{b}\}^{\mathrm{T}}\left[\boldsymbol{K}_{P}\right]\{\boldsymbol{b}\},
$$

where $\delta u_{y}\left(x_{P}, t\right)$ and $\delta u_{z}\left(x_{P}, t\right)$ are the virtual displacements at the thrust application point, and

$$
\left[\boldsymbol{K}_{P}\right]=P\left[\boldsymbol{\Phi}\left(x_{P}\right)\right]^{\mathrm{T}}\left[\boldsymbol{\Psi}\left(x_{P}\right)\right]
$$

Aerodynamic drag is split into concentrated drag force $F_{D_{1}}$ at the nose region and distributed drag $f_{D_{2}}(x, t)$ along the longitudinal axis of the missile. Axial aerodynamic force $F_{A x}$ is given by

$$
F_{A x}=-F_{D_{1}}-F_{D_{2}}=-F_{D_{1}}-\int_{l_{b}} f_{D_{2}}(x, t) \mathrm{d} x .
$$

The components $M_{D y}$ and $M_{D z}$ of moment that result from the concentrated drag force $F_{D_{1}}$ are

$$
\begin{gathered}
M_{D y}=-F_{D_{1}} u_{z}\left(x_{D}, t\right)-F_{D_{1}} x_{D} \theta_{y}\left(x_{D}, t\right), \\
M_{D z}=F_{D_{1}} u_{y}\left(x_{D}, t\right)-F_{D_{1}} x_{D} \theta_{z}\left(x_{D}, t\right),
\end{gathered}
$$

where $x_{D}$ is the axial position of the application point of the concentrated drag force $F_{D_{1}}$.

The virtual work of the concentrated drag force $F_{D_{1}}$ is

$$
\delta W_{D}=-F_{D_{1}} \theta_{z}\left(x_{D}, t\right) \delta u_{y}\left(x_{D}, t\right)+F_{D_{1}} \theta_{y}\left(x_{D}, t\right) \delta u_{z}\left(x_{D}, t\right)=\{\delta \boldsymbol{a}\}^{\mathrm{T}}\left[\boldsymbol{K}_{D}\right]\{\boldsymbol{a}\}+\{\delta \boldsymbol{b}\}^{\mathrm{T}}\left[\boldsymbol{K}_{D}\right]\{\boldsymbol{b}\},
$$

where

$$
\left[\boldsymbol{K}_{D}\right]=-F_{D_{1}}\left[\boldsymbol{\Phi}\left(x_{D}\right)\right]^{\mathrm{T}}\left[\boldsymbol{\Psi}\left(x_{D}\right)\right] .
$$

Inside the missile, a compressive axial force is produced by the effects of thrust, drag, and inertia force. Although the axial deformation of the missile is not considered, transverse bending and shear deformation can produce axial displacement; therefore, the axial force can do work.

As shown in Fig. $4, P_{N}(x, t)$ is the distributed axial force for the arbitrary position $x$ based on the force equilibrium equation obtained by

$$
P_{N}=\left(1-\frac{m_{x}}{m_{b}}\right) P+\frac{m_{x}}{m_{b}} F_{D_{1}}+\left(\frac{m_{x}}{m_{b}} F_{D_{2}}-F_{D x}\right),
$$

where $m_{x}=\int_{x_{P}}^{x} \rho A \mathrm{~d} x, F_{D x}=\int_{x_{P}}^{x} f_{D_{2}}(x, t) \mathrm{d} x$. 


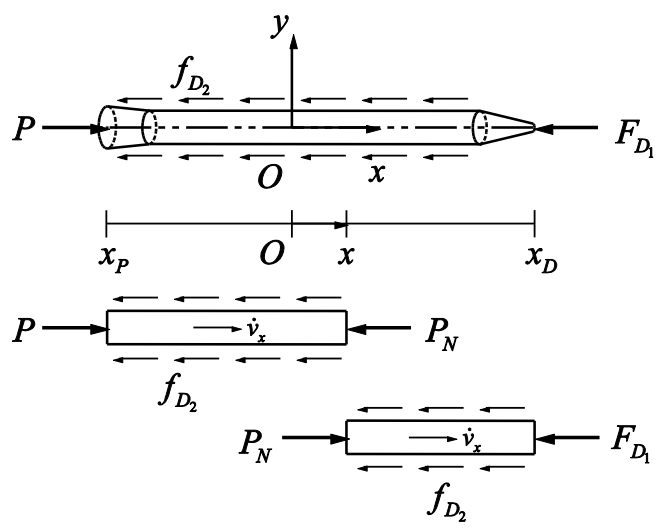

Fig. 4 Distributed axial force.

The effects of the axial displacement caused by the transverse bending and shear deformation [27] of the missile structure are considered, and the obtained axial displacement is

$$
\mathrm{d} u_{x}=-\frac{1}{2}\left[u_{y}^{\prime 2}-\left(\frac{E I}{\kappa G A}\right)^{2} u_{y}^{\prime \prime \prime 2}+u_{z}^{\prime 2}-\left(\frac{E I}{\kappa G A}\right)^{2} u_{z}^{\prime \prime \prime 2}\right] \mathrm{d} x .
$$

Given the compressive force of the axial force, the work of the axial force $P_{N}$ is

$$
W_{P_{N}}=\frac{1}{2} \int_{l_{b}} P_{N}\left[u_{y}^{\prime 2}-\left(\frac{E I}{\kappa G A}\right)^{2} u_{y}^{\prime \prime \prime 2}+u_{z}^{\prime 2}-\left(\frac{E I}{\kappa G A}\right)^{2} u_{z}^{\prime \prime \prime 2}\right] \mathrm{d} x=\frac{1}{2}\{\mathbf{a}\}^{\mathrm{T}}\left[\mathbf{K}_{P_{N}}\right]\{\mathbf{a}\}+\frac{1}{2}\{\mathbf{b}\}^{\mathrm{T}}\left[\mathbf{K}_{P_{N}}\right]\{\mathbf{b}\},
$$

where

$$
\left[\boldsymbol{K}_{P_{N}}\right]=\int_{l_{b}} P_{N}\left[\left[\boldsymbol{\Phi}^{\prime}\right]^{\mathrm{T}}\left[\boldsymbol{\Phi}^{\prime}\right]-\left(\frac{E I}{\kappa G A}\right)^{2}\left[\boldsymbol{\Phi}^{\prime \prime \prime}\right]^{\mathrm{T}}\left[\boldsymbol{\Phi}^{\prime \prime \prime}\right]\right] \mathrm{d} x,
$$

\subsection{Work of lateral aerodynamic forces}

The definition of angle of attack $\alpha$ and angle of sideslip $\beta$ is adopted by corresponding angle relationship between quasi-speed coordinate system and quasi-body coordinate system. Using quasi-steady theory of slender, local effective angle of attack $\alpha$ is considered, which is composed of angle of attack $\alpha_{0}$ of rigid missile, the bending slope $u_{z}^{\prime}$, the structure deflection rate $\dot{u}_{z}$, angular rate $\omega_{0 y}$, and spinning speed $\Omega[16,28]$.

$$
\alpha(x, t)=\alpha_{0}-u_{z}^{\prime}(x, t)+\frac{1}{v_{x}} \dot{u}_{z}(x, t)-\frac{\omega_{0 y}}{v_{x}} x+\frac{\Omega}{v_{x}} u_{y}(x, t) .
$$

Similarly, the angle of sideslip $\beta$ can be expressed as

$$
\beta(x, t)=\beta_{0}-u_{y}^{\prime}(x, t)+\frac{1}{v_{x}} \dot{u}_{y}(x, t)+\frac{\omega_{0 z}}{v_{x}} x-\frac{\Omega}{v_{x}} u_{z}(x, t) .
$$

For small angles, angle of attack and sideslip of rigid missile can be written as 


$$
\alpha_{0}=\frac{v_{z}}{v_{x}}, \beta_{0}=\frac{v_{y}}{v_{x}} .
$$

Lateral aerodynamic force distributions of the missile are

$$
\begin{aligned}
& f_{A y}(x, t)=-l_{\beta}(x) \beta(x, t)=-\beta_{0} l_{\beta}(x)+l_{\beta}(x)\left[\boldsymbol{\Phi}^{\prime}\right]\{\boldsymbol{a}\}-\frac{1}{v_{x}} l_{\beta}(x)[\boldsymbol{\Phi}]\{\dot{\boldsymbol{a}}\}-\frac{\omega_{0 z}}{v_{x}} x l_{\beta}(x)+\frac{\Omega}{v_{x}} l_{\beta}(x)[\boldsymbol{\Phi}]\{\boldsymbol{b}\}, \\
& f_{A z}(x, t)=-l_{\alpha}(x) \alpha(x, t)=-\alpha_{0} l_{\alpha}(x)+l_{\alpha}(x)\left[\boldsymbol{\Phi}^{\prime}\right]\{\boldsymbol{b}\}-\frac{1}{v_{x}} l_{\alpha}(x)[\boldsymbol{\Phi}]\{\dot{\boldsymbol{b}}\}+\frac{\omega_{0 y}}{v_{x}} x l_{\alpha}(x)-\frac{\Omega}{v_{x}} l_{\alpha}(x)[\boldsymbol{\Phi}]\{\boldsymbol{a}\},
\end{aligned}
$$

where $l_{\alpha}(x)$ and $l_{\beta}(x)$ is lift and side force derivative per unit length, respectively. Given the assumption that the missile has axisymmetric structures, is $l_{\alpha}(x)=l_{\beta}(x)$ obtained.

The work conducted because of lateral aerodynamic forces $f_{A y}(x, t)$ and $f_{A z}(x, t)$ on virtual displacements $\delta u_{y}(x, t)$ and $\delta u_{z}(x, t)$ can be written as

$$
\delta W_{A}=\int_{l_{b}} f_{A y}(x, t) \delta u_{y}(x, t) \mathrm{d} x+\int_{l_{b}} f_{A z}(x, t) \delta u_{z}(x, t) \mathrm{d} x=\{\delta \boldsymbol{a}\}^{\mathrm{T}}\left\{\boldsymbol{Q}_{A y}\right\}+\{\delta \boldsymbol{b}\}^{\mathrm{T}}\left\{\boldsymbol{Q}_{A z}\right\},
$$

where

$$
\begin{aligned}
& \left\{\boldsymbol{Q}_{A y}\right\}=-\beta_{0}\left\{\boldsymbol{I}_{1}\right\}+\left[\boldsymbol{I}_{2}\right]\{\boldsymbol{a}\}-\frac{1}{v_{x}}\left[\boldsymbol{I}_{3}\right]\{\dot{\boldsymbol{a}}\}-\frac{\omega_{0 z}}{v_{x}}\left\{\boldsymbol{I}_{4}\right\}+\frac{\Omega}{v_{x}}\left[\boldsymbol{I}_{3}\right]\{\boldsymbol{b}\}, \\
& \left\{\boldsymbol{Q}_{A z}\right\}=-\alpha_{0}\left\{\boldsymbol{I}_{1}\right\}+\left[\boldsymbol{I}_{2}\right]\{\boldsymbol{b}\}-\frac{1}{v_{x}}\left[\boldsymbol{I}_{3}\right]\{\dot{\boldsymbol{b}}\}+\frac{\omega_{0 y}}{v_{x}}\left\{\boldsymbol{I}_{4}\right\}-\frac{\Omega}{v_{x}}\left[\boldsymbol{I}_{3}\right]\{\boldsymbol{a}\} .
\end{aligned}
$$

Lateral aerodynamic forces are obtained as

$$
\begin{aligned}
& F_{A y}=-\int_{l_{b}} l_{\beta}(x) \beta(x, t) \mathrm{d} x=-I_{5} \beta_{0}+\left[\boldsymbol{I}_{6}\right]\{\boldsymbol{a}\}-\frac{1}{v_{x}}\left[\boldsymbol{I}_{7}\right]\{\dot{\boldsymbol{a}}\}-I_{8} \frac{\omega_{0 z}}{v_{x}}+\frac{\Omega}{v_{x}}\left[\boldsymbol{I}_{7}\right]\{\boldsymbol{b}\}, \\
& F_{A z}=-\int_{l_{b}} l_{\alpha}(x) \alpha(x, t) \mathrm{d} x=-I_{5} \alpha_{0}+\left[\boldsymbol{I}_{6}\right]\{\boldsymbol{b}\}-\frac{1}{v_{x}}\left[\boldsymbol{I}_{7}\right]\{\dot{\boldsymbol{b}}\}+I_{8} \frac{\omega_{0 y}}{v_{x}}-\frac{\Omega}{v_{x}}\left[\boldsymbol{I}_{7}\right]\{\boldsymbol{a}\} .
\end{aligned}
$$

Aerodynamic moments are obtained as

$$
\begin{gathered}
M_{A y}=\int_{l_{b}} x l_{\alpha}(x) \alpha(x, t) \mathrm{d} x=I_{8} \alpha_{0}-\left[\boldsymbol{I}_{9}\right]\{\boldsymbol{b}\}+\frac{1}{v_{x}}\left[\boldsymbol{I}_{10}\right]\{\dot{\boldsymbol{b}}\}-\frac{\omega_{0 y}}{v_{x}} I_{11}+\frac{\Omega}{v_{x}}\left[\boldsymbol{I}_{10}\right]\{\boldsymbol{a}\}, \\
M_{A z}=-\int_{l_{b}} x l_{\beta}(x) \beta(x, t) \mathrm{d} x=-I_{8} \beta_{0}+\left[\boldsymbol{I}_{9}\right]\{\boldsymbol{a}\}-\frac{1}{v_{x}}\left[\boldsymbol{I}_{10}\right]\{\dot{\boldsymbol{a}}\}-\frac{\omega_{0 z}}{v_{x}} I_{11}+\frac{\Omega}{v_{x}}\left[\boldsymbol{I}_{10}\right]\{\boldsymbol{b}\},
\end{gathered}
$$

where coefficients $C_{N \alpha}, C_{M \alpha}$, and $\left[\boldsymbol{I}_{i}\right]$ are defined in Appendix A.

\subsection{Equations of motion}

Perform the indicated differentiations in Lagrange's equation of nonconservative system with generalized coordinates $a_{i}(t)$ and $b_{i}(t)$, respectively, obtained elastic vibration equations of spinning missile are 


$$
\begin{gathered}
{[\boldsymbol{M}]\{\ddot{\boldsymbol{a}}\}+\left[\boldsymbol{C}_{D}\right]\{\dot{\boldsymbol{a}}\}+\Omega\left[\boldsymbol{J}_{p}\right]\{\dot{\boldsymbol{b}}\}+\left([\boldsymbol{K}]-\left[\boldsymbol{K}_{P_{N}}\right]-\left[\boldsymbol{K}_{P}\right]-\left[\boldsymbol{K}_{D}\right]\right)\{\boldsymbol{a}\}-\left(\omega_{0 x}^{2}[\boldsymbol{M}]+\omega_{0 y}^{2}\left[\boldsymbol{J}_{p}\right]+\omega_{0 z}^{2}\left[\boldsymbol{M}_{T}\right]\right)\{\boldsymbol{a}\}} \\
+\left(2 \dot{\Omega}+\dot{\omega}_{0 x}\right)\left[\boldsymbol{M}_{R}\right]\{\boldsymbol{b}\}+\omega_{0 y} \omega_{0 z}\left(\left[\boldsymbol{M}_{T}\right]-\left[\boldsymbol{M}_{R}\right]\right)\{\boldsymbol{b}\}-\left(\omega_{0 x} \omega_{0 y}+2 \omega_{0 y} \Omega-\dot{\omega}_{0 z}\right)\left[\boldsymbol{I}_{0}\right]^{\mathrm{T}}=\left\{\boldsymbol{Q}_{A y}\right\}+\left\{\boldsymbol{Q}_{B y}\right\}, \\
{[\boldsymbol{M}]\{\ddot{\boldsymbol{b}}\}+\left[\boldsymbol{C}_{D}\right]\{\dot{\boldsymbol{b}}\}-\Omega\left[\boldsymbol{J}_{p}\right]\{\dot{\boldsymbol{a}}\}+\left([\boldsymbol{K}]-\left[\boldsymbol{K}_{P_{N}}\right]-\left[\boldsymbol{K}_{P}\right]-\left[\boldsymbol{K}_{D}\right]\right)\{\boldsymbol{b}\}-\left(\omega_{0 x}^{2}[\boldsymbol{M}]+\omega_{0 y}^{2}\left[\boldsymbol{M}_{T}\right]+\omega_{0 z}^{2}\left[\boldsymbol{J}_{p}\right]\right)\{\boldsymbol{b}\}} \\
+\dot{\omega}_{0 x}\left[\boldsymbol{M}_{R}\right]\{\boldsymbol{a}\}+\omega_{0 y} \omega_{0 z}\left(\left[\boldsymbol{M}_{T}\right]-\left[\boldsymbol{M}_{R}\right]\right)\{\boldsymbol{a}\}-\left(\omega_{0 x} \omega_{0 z}+2 \omega_{0 z} \Omega+\dot{\omega}_{0 y}\right)\left[\boldsymbol{I}_{0}\right]^{\mathrm{T}}=\left\{\boldsymbol{Q}_{A_{z}}\right\}+\left\{\boldsymbol{Q}_{B z}\right\},
\end{gathered}
$$

where $\left\{\boldsymbol{Q}_{B y}\right\}$ and $\left\{\boldsymbol{Q}_{B z}\right\}$ are other generalized forces on the missile in addition to the aerodynamic forces.

According to the Lagrange's equations in floating coordinate system [29, 30], angular rate equations of spinning missile are obtained as

$$
\begin{aligned}
J_{x}\left(\dot{\omega}_{0 x}+\dot{\Omega}\right)+\{\ddot{\boldsymbol{a}}\}^{\mathrm{T}}\left[\boldsymbol{M}_{R}\right]\{\boldsymbol{b}\}+2 \omega_{0 x}\left(\{\boldsymbol{a}\}^{\mathrm{T}}[\boldsymbol{M}]\{\dot{\boldsymbol{a}}\}+\{\boldsymbol{b}\}^{\mathrm{T}}[\boldsymbol{M}]\{\dot{\boldsymbol{b}}\}\right)+\{\boldsymbol{a}\}^{\mathrm{T}}\left[\dot{\omega}_{0 x}[\boldsymbol{M}]+\omega_{0 y} \omega_{0 z}\left(\left[\boldsymbol{M}_{T}\right]-\left[\boldsymbol{J}_{p}\right]\right)\right]\{\boldsymbol{a}\} \\
+2\{\dot{\boldsymbol{a}}\}^{\mathrm{T}}\left[\boldsymbol{M}_{R}\right]\{\dot{\boldsymbol{b}}\}+\{\boldsymbol{b}\}^{\mathrm{T}}\left[\dot{\omega}_{0 x}[\boldsymbol{M}]+\omega_{0 y} \omega_{0 z}\left(\left[\boldsymbol{J}_{p}\right]-\left[\boldsymbol{M}_{T}\right]\right)\right]\{\boldsymbol{b}\}+\{\boldsymbol{a}\}^{\mathrm{T}}\left[\boldsymbol{M}_{R}\right]\{\dot{\boldsymbol{b}}\} \\
+\left(\omega_{0 y}^{2}-\omega_{0 z}^{2}\right)\{\boldsymbol{a}\}^{\mathrm{T}}\left(\left[\boldsymbol{M}_{R}\right]-\left[\boldsymbol{M}_{T}\right]\right)\{\boldsymbol{b}\}+\left(\dot{\omega}_{0 y}-\omega_{0 x} \omega_{0 z}-2 \omega_{0 z} \Omega\right)\left[\boldsymbol{I}_{0}\right]\{\boldsymbol{a}\} \\
+\left(\dot{\omega}_{0 z}+\omega_{0 x} \omega_{0 y}+2 \omega_{0 y} \Omega\right)\left[\boldsymbol{I}_{0}\right]\{\boldsymbol{b}\}+2 \omega_{0 y}\left[\boldsymbol{I}_{0}\right]\{\dot{\boldsymbol{a}}\}+2 \omega_{0 z}\left[\boldsymbol{I}_{0}\right]\{\dot{\boldsymbol{b}}\}=M_{A x}+M_{B x}, \\
\frac{1}{2} J_{x}\left(\dot{\omega}_{0 y}+\omega_{0 x} \omega_{0 z}+2 \omega_{0 z} \Omega\right)+J_{y}\left(\dot{\omega}_{0 y}-\omega_{0 x} \omega_{0 z}\right)+2 \omega_{0 y}\{\boldsymbol{a}\}^{\mathrm{T}}\left[\boldsymbol{J}_{p}\right]\{\dot{\boldsymbol{a}}\}+\left(2 \dot{\omega}_{0 y}+\omega_{0 x} \omega_{0 z}\right)\{\boldsymbol{a}\}^{\mathrm{T}}\left[\boldsymbol{M}_{R}\right]\{\boldsymbol{a}\} \\
+2 \omega_{0 y}\{\boldsymbol{b}\}^{\mathrm{T}}\left[\boldsymbol{M}_{T}\right]\{\dot{\boldsymbol{b}}\}+\{\boldsymbol{b}\}^{\mathrm{T}}\left[\dot{\omega}_{0 y}\left[\boldsymbol{M}_{T}\right]+\omega_{0 x} \omega_{0 z}\left([\boldsymbol{M}]-\left[\boldsymbol{J}_{p}\right]\right)\right]\{\boldsymbol{b}\}+\omega_{0 z}\{\dot{\boldsymbol{a}}\}^{\mathrm{T}}\left(\left[\boldsymbol{J}_{p}\right]-\left[\boldsymbol{M}_{T}\right]\right)\{\boldsymbol{b}\} \\
+\omega_{0 z}\{\boldsymbol{a}\}^{\mathrm{T}}\left(\left[\boldsymbol{J}_{p}\right]-\left[\boldsymbol{M}_{T}\right]\right)\{\dot{\boldsymbol{b}}\}+\left(\dot{\omega}_{0 z}-\omega_{0 x} \omega_{0 y}\right)\{\boldsymbol{a}\}^{\mathrm{T}}\left(\left[\boldsymbol{M}_{R}\right]-\left[\boldsymbol{M}_{T}\right]\right)\{\boldsymbol{b}\}-\left[\boldsymbol{I}_{0}\right]\{\ddot{\boldsymbol{b}}\}+2 \Omega\left[\boldsymbol{I}_{0}\right]\{\dot{\boldsymbol{a}}\} \\
+\left(\dot{\omega}_{0 x}+2 \dot{\Omega}+\omega_{0 y} \omega_{0 z}\right)\left[\boldsymbol{I}_{0}\right]\{\boldsymbol{a}\}+\left(\omega_{0 z}^{2}-\omega_{0 x}^{2}-2 \omega_{0 x} \Omega\right)\left[\boldsymbol{I}_{0}\right]\{\boldsymbol{b}\}=M_{A y}+M_{B y} \\
\frac{1}{2} J_{x}\left(\dot{\omega}_{0 z}-\omega_{0 x} \omega_{0 y}-2 \omega_{0 y} \Omega\right)+J_{y}\left(\dot{\omega}_{0 z}+\omega_{0 x} \omega_{0 y}\right)+2 \omega_{0 z}\{\boldsymbol{b}\}^{\mathrm{T}}\left[\boldsymbol{J}_{p}\right]\{\dot{\boldsymbol{b}}\}+\left(2 \dot{\omega}_{0 z}-\omega_{0 x} \omega_{0 y}\right)\{\boldsymbol{b}\}^{\mathrm{T}}\left[\boldsymbol{M}_{R}\right]\{\boldsymbol{b}\} \\
+2 \omega_{0 z}\{\boldsymbol{a}\}^{\mathrm{T}}\left[\boldsymbol{M}_{T}\right]\{\dot{\boldsymbol{a}}\}+\{\boldsymbol{a}\}^{\mathrm{T}}\left[\dot{\omega}_{0 z}\left[\boldsymbol{M}_{T}\right]+\omega_{0 x} \omega_{0 y}\left(\left[\boldsymbol{J}_{p}\right]-[\boldsymbol{M}]\right)\right]\{\boldsymbol{a}\}-\omega_{0 y}\{\dot{\boldsymbol{a}}\}^{\mathrm{T}}\left[\boldsymbol{M}_{T}\right]\{\boldsymbol{b}\} \\
-\omega_{0 y}\{\boldsymbol{a}\}^{\mathrm{T}}\left[\boldsymbol{M}_{T}\right]\{\dot{\boldsymbol{b}}\}+\left(\dot{\omega}_{0 y}+\omega_{0 x} \omega_{0 z}\right)\{\boldsymbol{a}\}^{\mathrm{T}}\left(\left[\boldsymbol{M}_{R}\right]-\left[\boldsymbol{M}_{T}\right]\right)\{\boldsymbol{b}\}+\left[\boldsymbol{I}_{0}\right]\{\ddot{\boldsymbol{a}}\}+2 \Omega\left[\boldsymbol{I}_{0}\right]\{\dot{\boldsymbol{b}}\} \\
+\left(\dot{\omega}_{0 x}+2 \dot{\Omega}-\omega_{0 y} \omega_{0 z}\right)\left[\boldsymbol{I}_{0}\right]\{\boldsymbol{b}\}+\left(\omega_{0 x}^{2}-\omega_{0 y}^{2}+2 \omega_{0 x} \Omega\right)\left[\boldsymbol{I}_{0}\right]\{\boldsymbol{a}\}=M_{A z}+M_{B z},
\end{aligned}
$$

where $M_{A x}$ is aerodynamic moment about longitudinal direction of missile; $M_{B x}, M_{B y}$, and $M_{B z}$ are other moments on the missile in addition to aerodynamic moments.

According to the Lagrange's equations in floating coordinate system, translational equations of spinning missile are obtained as

$$
\begin{aligned}
& m_{b}\left(\dot{v}_{x}+\omega_{0 y} v_{z}-\omega_{0 z} v_{y}\right)=F_{G x}+P_{x}+F_{A x}+F_{B x}, \\
& m_{b}\left(\dot{v}_{y}+\omega_{0 z} v_{x}-\omega_{0 x} v_{z}\right)=F_{G y}+P_{y}+F_{A y}+F_{B y},
\end{aligned}
$$




$$
m_{b}\left(\dot{v}_{z}+\omega_{0 x} v_{y}-\omega_{0 y} v_{x}\right)=F_{G z}+P_{z}+F_{A z}+F_{B z}
$$

where $F_{G x}, F_{G y}$, and $F_{G z}$ are the components of the gravity force in the nonspinning coordinate system; and $F_{B x}$, $F_{B y}$, and $F_{B z}$ are the other forces acting on the missile.

Based on Eqs. (62-69) and the relationship between the rigid angular rates and attitude angles of the missile, i.e., Eq. (19), the general equations of motion of the spinning missile are obtained.

\section{Stability equation}

Equations of motion of flexible spinning missile contain nonlinear and coupling terms. The equation of motion is simplified to obtain the stability equation of missile. The same treatment as Ref. [1] is used and the longitudinal direction velocity $v_{x}$ and spinning speed $\Omega$ of missile are regarded as constants in the present study. Smaller rotational component $\omega_{0 x}$ and coupling items between various variables and high order nonlinear terms are ignored, which makes the equation linearization. The complex variables are defined as

$$
\begin{gathered}
\{\boldsymbol{q}\}=\{\boldsymbol{a}\}+\mathrm{i}\{\boldsymbol{b}\}, \\
\omega_{0}=\omega_{0 y}+\mathrm{i} \omega_{0 z}, \\
\delta=\beta_{0}+\mathrm{i} \alpha_{0} .
\end{gathered}
$$

The equations of lateral motion using complex variables can be written as

$$
[\boldsymbol{A}]\left\{\begin{array}{c}
\ddot{\omega}_{0} \\
\ddot{\delta} \\
\{\ddot{\boldsymbol{q}}\}
\end{array}\right\}+[\boldsymbol{B}]\left\{\begin{array}{c}
\dot{\omega}_{0} \\
\dot{\delta} \\
\{\dot{\boldsymbol{q}}\}
\end{array}\right\}+[\boldsymbol{C}]\left\{\begin{array}{c}
\omega_{0} \\
\delta \\
\{\boldsymbol{q}\}
\end{array}\right\}=\{\boldsymbol{F}\},
$$

where

$$
\begin{gathered}
{[\boldsymbol{A}]=\left[\begin{array}{ccc}
0 & 0 & \mathrm{i}\left[\boldsymbol{I}_{0}\right] \\
0 & 0 & \mathbf{0}_{1 \times m} \\
\mathbf{0}_{m \times 1} & \mathbf{0}_{m \times 1} & {[\boldsymbol{M}]}
\end{array}\right],} \\
{[\boldsymbol{B}]=\left[\begin{array}{ccc}
\left(\frac{1}{2} J_{x}+J_{y}\right) & 0 & \left(\mathrm{i} \frac{1}{v_{x}}\left[\boldsymbol{I}_{10}\right]+2 \Omega\left[\boldsymbol{I}_{0}\right]\right) \\
0 & m_{b} v_{x} & \frac{1}{v_{x}}\left[\boldsymbol{I}_{7}\right] \\
\mathbf{0}_{m \times 1} & \mathbf{0}_{m \times 1} & \left(\left[\boldsymbol{C}_{D}\right]-\mathrm{i} \Omega\left[\boldsymbol{J}_{p}\right]+\frac{1}{v_{x}}\left[\boldsymbol{I}_{3}\right]\right)
\end{array}\right],}
\end{gathered}
$$




$$
[\boldsymbol{C}]=\left[\begin{array}{ccc}
\frac{1}{v_{x}} I_{11}-\mathrm{i} J_{x} \Omega & \mathrm{i} I_{8} & {\left[-\left(\mathrm{i}\left[\boldsymbol{I}_{9}\right]+\Omega\left[\boldsymbol{I}_{10}\right] / v_{x}\right)+\mathrm{i} P\left(\left[\boldsymbol{\Phi}\left(x_{P}\right)\right]-x_{P}\left[\boldsymbol{\Psi}\left(x_{P}\right)\right]\right)\right.} \\
-\mathrm{i} \frac{1}{v_{x}} I_{8}-\mathrm{i} m_{b} v_{x} & I_{5} & \left.-\mathrm{i} F_{D_{1}}\left(\left[\boldsymbol{\Phi}\left(x_{D}\right)\right]-x_{D}\left[\boldsymbol{\Psi}\left(x_{D}\right)\right]\right)\right] \\
-2 \Omega\left[\boldsymbol{I}_{0}\right]^{\mathrm{T}}-\mathrm{i} \frac{1}{v_{x}}\left\{\boldsymbol{I}_{4}\right\} & \left\{\boldsymbol{I}_{1}\right\} & {[\boldsymbol{K}]-\left[\boldsymbol{K}_{P_{N}}\right]-\left[\boldsymbol{K}_{P}\right]-\left[\boldsymbol{K}_{D}\right]-\left[\boldsymbol{I}_{2}\right]+\mathrm{i} \frac{\Omega}{v_{x}}\left[\boldsymbol{I}_{3}\right]}
\end{array}\right],
$$

where the coefficient matrices $[\boldsymbol{A}],[\boldsymbol{B}]$, and $[\boldsymbol{C}]$ are the functions about dynamic pressure and the spinning speed.

Converting Eq. (71) to Laplace transform, stability equation of the spinning missile is obtained as

$$
\left|[\boldsymbol{A}] s^{2}+[\boldsymbol{B}] s+[\boldsymbol{C}]\right|=0 .
$$

Stability of motion of missile is determined by characteristic equation as shown in Eq. (78). According to the properties of the complex root $s$, the stability of system is estimated. Dynamic stability requires that all roots $s=\sigma+\mathrm{i} \omega$ have negative real parts. Instability will occur when at least one of the roots has positive real part. For static stability, setting $s=0$, determined by determinant $|[\boldsymbol{C}]|=0$, the solved dynamic pressure $q$ is divergence dynamic pressure $q_{\text {div }}$ of missile.

\section{Stability calculations}

A finned spinning missile with fineness ratio of 25 is taken as an example. The parameters of the missile are shown in Table 1. The missile used the non-uniform beam model, mass distribution, rotational inertia distribution and stiffness distribution of the missile are shown in Figs. 5-7, respectively. The first order mode shape is shown in Fig. 8. Lateral aerodynamic coefficient derivative distribution along longitudinal axis of missile is shown in Fig. 9.

Table 1 Parameters of missile

\begin{tabular}{cc}
\hline Parameter & Value \\
\hline$m_{b}$ & $850 \mathrm{~kg}$ \\
$l_{b}$ & $7.5 \mathrm{~m}$ \\
$d$ & $0.3 \mathrm{~m}$ \\
$J_{x}$ & $18.50 \mathrm{~kg} \cdot \mathrm{m}^{2}$ \\
$J_{y}$ & $3264.75 \mathrm{~kg} \cdot \mathrm{m}^{2}$ \\
$v_{x}$ & $1020 \mathrm{~m} \cdot \mathrm{s}^{-1}$ \\
$\alpha_{0}$ & $3^{\circ}$ \\
\hline
\end{tabular}




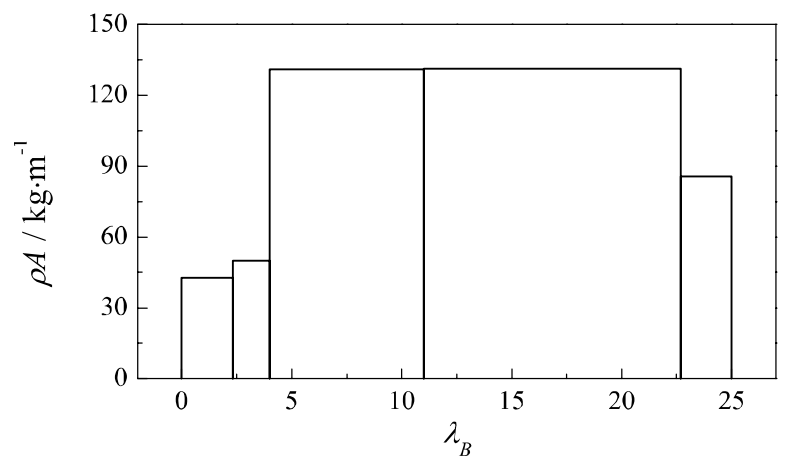

Fig. 5 Mass distribution of the missile along the longitudinal axis.

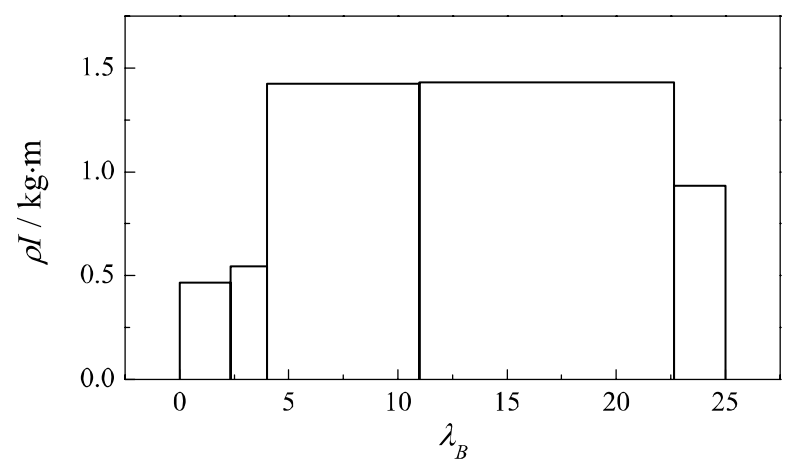

Fig. 6 Rotational inertia distribution of the missile along the longitudinal axis.

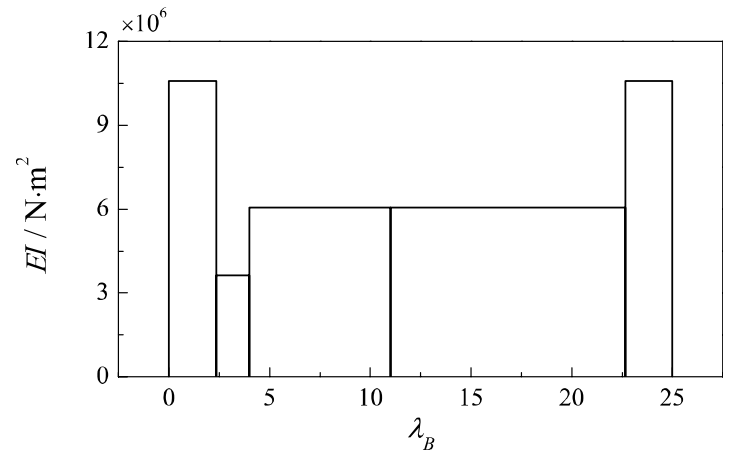

Fig. 7 Stiffness distribution of the missile along the longitudinal axis.

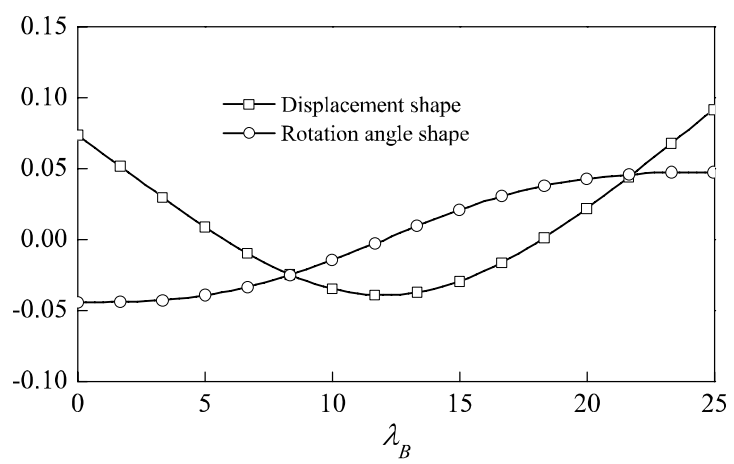

Fig. 8 First order mode shape of missile. 


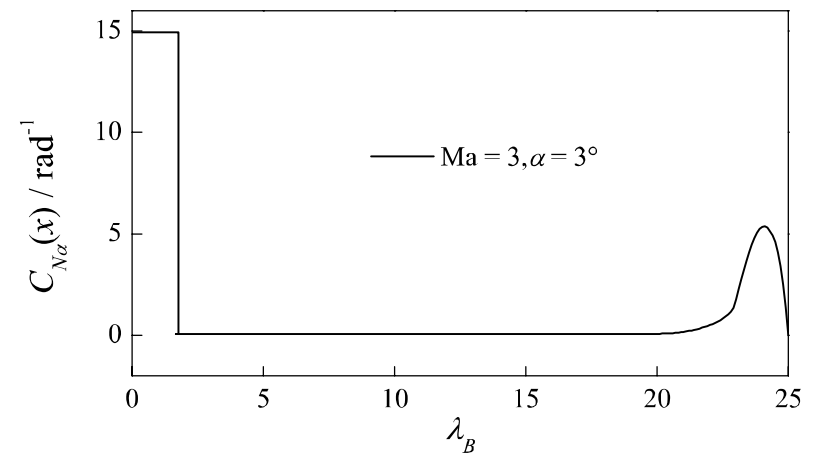

Fig. 9 Lateral aerodynamic coefficient derivative distribution along longitudinal axis of missile.

The results of static stability under different thrusts are shown in Fig. 10. The relative thrust is $\bar{P}=P / P_{\text {ref }}$; the reference thrust is $P_{\text {ref }}=680 \mathrm{kN} ; \bar{q}$ is the relative dynamic pressure, i.e., $\bar{q}=q / q_{\text {ref }}$; and $q_{\text {ref }}$ is the dynamic pressure values at $\mathrm{Ma}=3$. The value of $\lg \mid[\boldsymbol{C}]$ is used to judge the static stability of the missile [31]. When the value of $\lg [[\boldsymbol{C}] \mid$ suddenly decreases, the dynamic pressure reaches the value of the divergence dynamic pressure. The system appears to exhibit static instability, and thus, bending divergence is produced.

Based on the results in Fig. 10(a), when $\Omega=0$ rps, the divergence dynamic pressure decreases with the increase in thrust because thrust produces a compressive axial force and reduces structure stiffness. Aerodynamic force can easily cause a structure to produce elastic bending deformation. Thus, static instability is easy to achieve. When $\bar{P}=2.54$, the divergence dynamic pressure $\bar{q}_{\text {div }}=1$, which indicates that the reference dynamic pressure has already reached the current divergence dynamic pressure. As shown in Fig. 10(b), when spin occurs, changes in $\lg |[\boldsymbol{C}]|$ near the divergence dynamic pressure are flattened out. Under the same spinning speed, the change in amplitude of $\lg |[\boldsymbol{C}]|$ and the effect of spinning are more obvious when thrust is smaller. This phenomenon proves that the spin of the missile is beneficial for static stability.

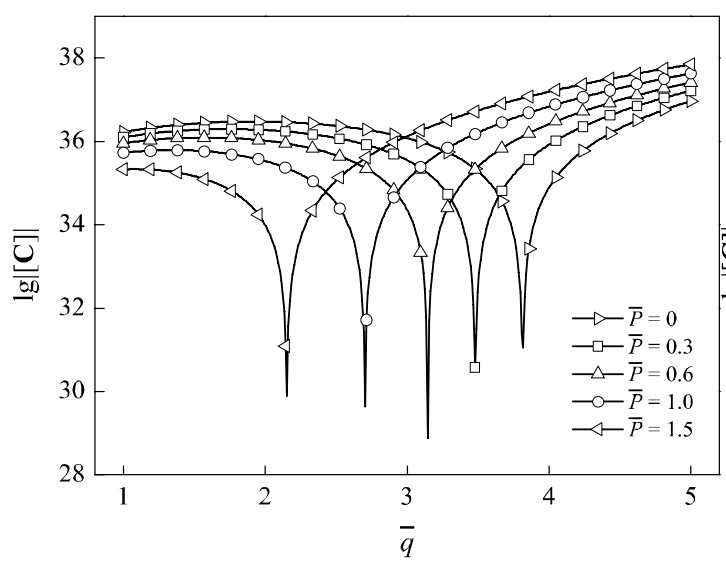

(a) $\Omega=0 \mathrm{rps}$

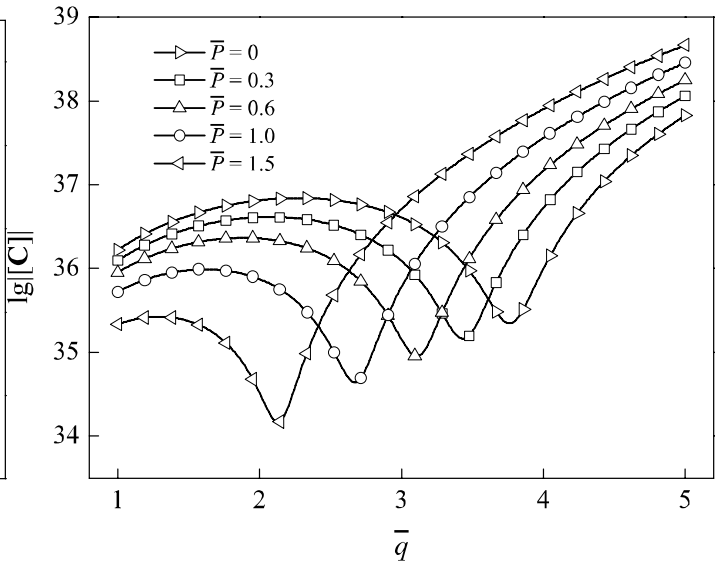

(b) $\Omega=10 \mathrm{rps}$

Fig. 10 Static stability of the spinning missile under different thrusts. 
The dynamic stability of the missile is considered under different thrusts, and the result is shown in Fig. 11, where $\omega_{F 1}$ and $\omega_{B 1}$ are the first-order forward and backward precession frequencies of the elastic spinning missile, and $\omega_{R F}$ and $\omega_{R B}$ are the rigid forward and backward precession frequencies of the missile (i.e., pitching or yawing motion frequencies). When $\bar{P}=2.26$, as shown in Fig. 11(b), the real parts of $\omega_{F 1}$ and $\omega_{R F}$ are simultaneously changed from negative to positive with the increase in spinning speed. Thus, thrust $\bar{P}=2.26$ is a critical value. When thrust is less than the critical value, as shown in Fig. 11(a), dynamic instability is produced by the elastic frequency, which shows that the structure exhibits flutter instability. When thrust is greater than the critical value, as shown in Fig. 11(c), dynamic instability is produced by the rigid frequency, which shows the rapid divergence of the rigid rotation speed of the missile. In addition, the spinning speed of dynamic instability initially increases and then decreases with the increase in thrust, and the turning point occurs at the critical thrust value.

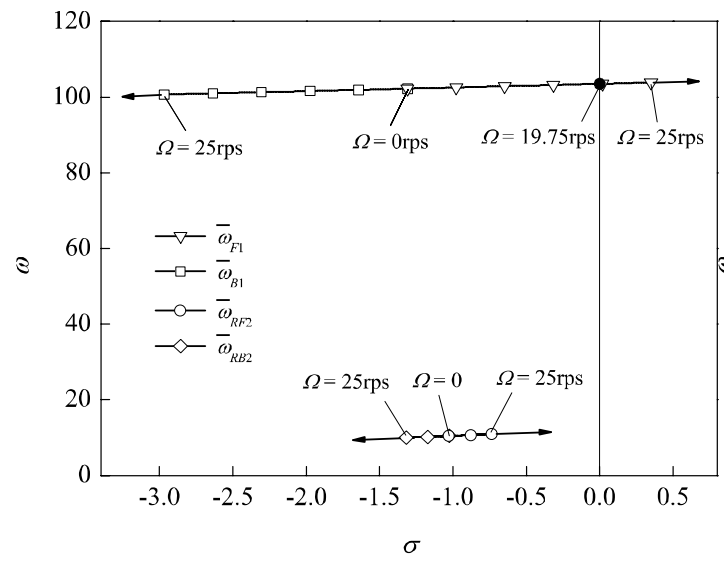

(a) $\bar{P}=1$

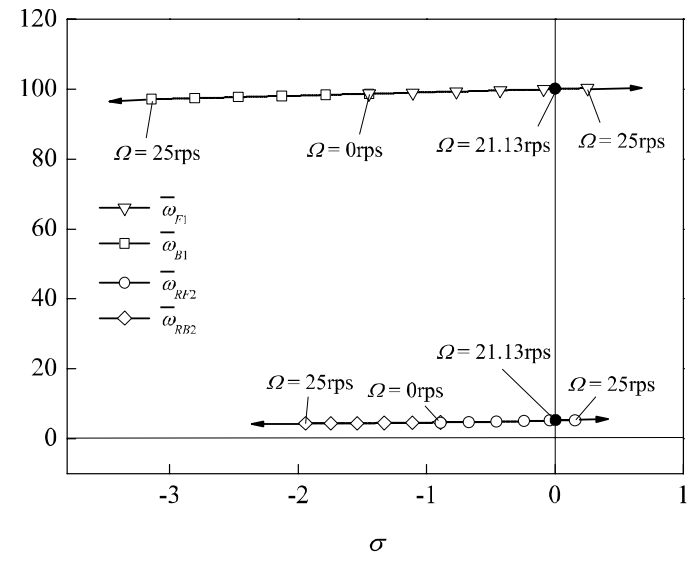

(b) $\bar{P}=2.26$

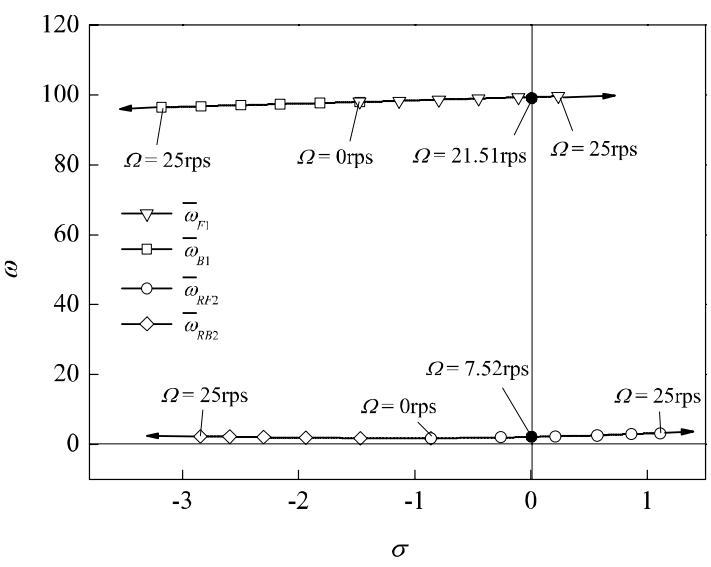

(c) $\bar{P}=2.5$

Fig. 11 Trajectories of complex roots under different thrusts.

\section{Dynamic responses calculation}

Acceleration terms in the general equations of motion of spinning missile are properly simplified, as shown in Eqs. (62-69). Available integral is solved by Runge-Kutta method at given initial conditions. 
Two cases are analyzed to verify static stability and dynamic stability under thrust. In Case 1, transient vibration responses are calculated at $\bar{P}=2.54, \mathrm{Ma}=3, \Omega=0 \mathrm{rps}$, and initial angle of attack $\alpha_{0}=3^{\circ}$. The results are shown in Fig. 12. In Case 2, the unbalanced responses under mass eccentric force are calculated at $\bar{P}=2.5, \mathrm{Ma}=3$, $\Omega=10 \mathrm{rps}$, initial angle of attack $\alpha_{0}=3^{\circ}$, and eccentricity of the center of mass $=1 \mathrm{~mm}$. The results are presented in Fig. 13. The calculation results show that the elastic displacements and angle of attack of the rigid missile spread rapidly under thrust and aerodynamic force in both cases because the missiles have reached their respective instability conditions. The dynamic pressure has reached the current divergence dynamic pressure in Case 1, and the spinning speed exceeds the current spinning speed of instability ( $\Omega=7.52 \mathrm{rps}$ ). The sustained action of thrust further increases dynamic pressure and accelerates the instability of the responses. The results of these two cases are consistent with those of the stability analysis.

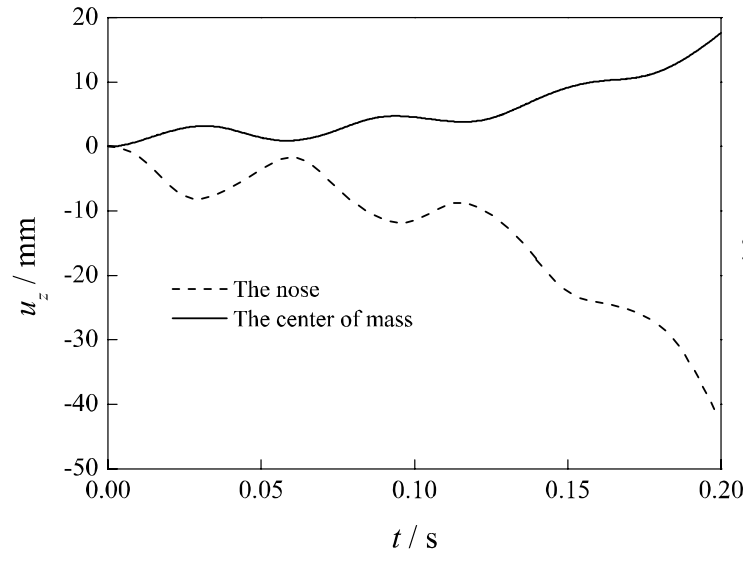

(a) Displacements of the nose and the center of mass

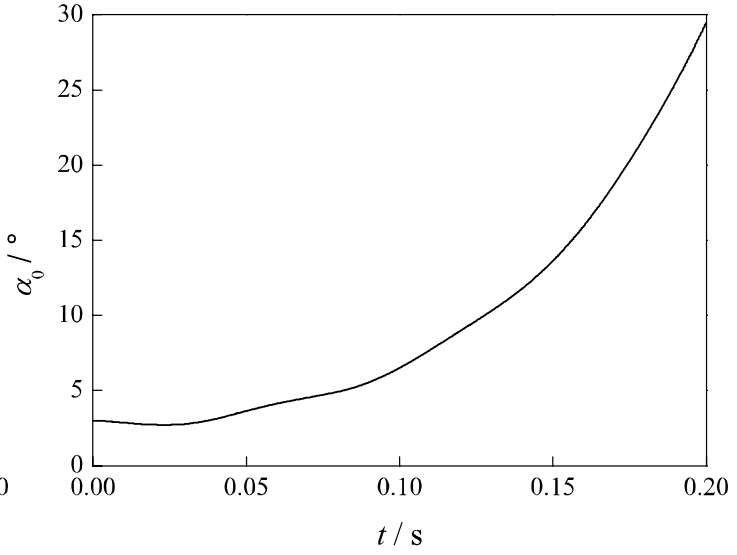

(b) Response of angle of attack of the rigid missile

Fig. 12 Responses at $\bar{P}=2.54, \mathrm{Ma}=3, \Omega=0 \mathrm{rps}$, and initial angle of attack $\alpha_{0}=3^{\circ}$.

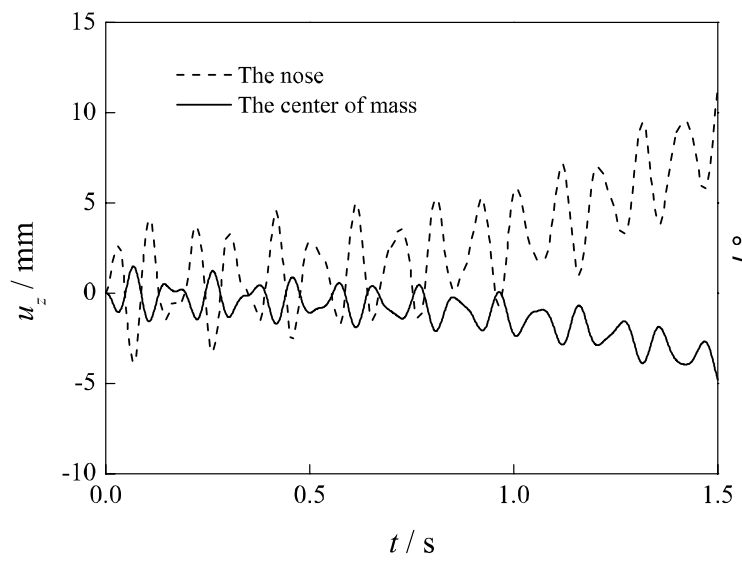

(a) Displacements of the nose and the center of mass

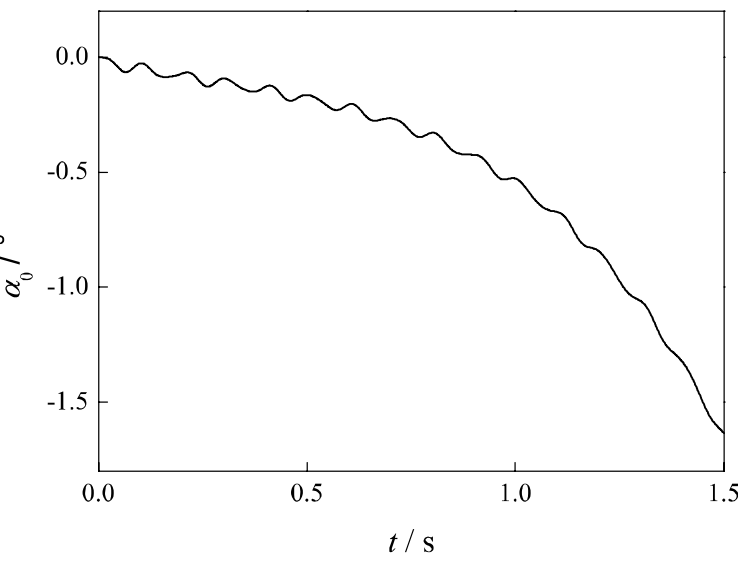

(b) Response of angle of attack of the rigid missile

Fig. 13 Unbalanced responses under mass eccentric force at $\bar{P}=2.5, \mathrm{Ma}=3, \Omega=10 \mathrm{rps}$, initial angle of attack $\alpha_{0}=3^{\circ}$, and eccentricity of the center of mass $=1 \mathrm{~mm}$. 


\section{Conclusions}

Considering the effect of follower thrust, the general equations of motion and stability of a flexible spinning missile in the powered flight phase are derived, and the stability of a typical spinning missile is calculated. The stability of the missile under different thrusts and spinning speeds is analyzed along with the numerical simulation dynamic response. According to the calculation results, the following conclusions are drawn:

(1) Thrust reduces structure stiffness, which facilitates static instability. Thus, thrust can decrease the divergent dynamic pressure of the spinning missile.

(2) Thrust has a critical value. When thrust is less than the critical value, dynamic instability is produced by the elastic frequency. When thrust is greater than the critical value, dynamic instability is produced by the rigid frequency. As thrust increases, the spinning speed of dynamic instability initially increases and then decreases, and the turning point occurs at the critical thrust value.

(3) The results of the calculation of transient vibration responses under thrust at different conditions are identical with the results of the stability analysis.

Thrust, spinning speed, and dynamic pressure have different influences on the stability of the spinning missile in the powered flight phase. Therefore, a reasonable design of thrust and spinning speed is necessary to avoid instability.

\section{Appendix A: Coefficients}

The aerodynamic coefficients and integral coefficients are

$$
\begin{aligned}
& \left\{\boldsymbol{I}_{1}\right\}=\int_{l_{b}} l_{\alpha}(x)[\boldsymbol{\Phi}]^{\mathrm{T}} \mathrm{d} x=\int_{l_{b}} l_{\beta}(x)[\boldsymbol{\Phi}]^{\mathrm{T}} \mathrm{d} x \\
& {\left[\boldsymbol{I}_{2}\right]=\int_{l_{b}} l_{\alpha}(x)[\boldsymbol{\Phi}]^{\mathrm{T}}\left[\boldsymbol{\Phi}^{\prime}\right] \mathrm{d} x=\int_{l_{b}} l_{\beta}(x)[\boldsymbol{\Phi}]^{\mathrm{T}}\left[\boldsymbol{\Phi}^{\prime}\right] \mathrm{d} x} \\
& {\left[\boldsymbol{I}_{3}\right]=\int_{l_{b}} l_{\alpha}(x)[\boldsymbol{\Phi}]^{\mathrm{T}}[\boldsymbol{\Phi}] \mathrm{d} x=\int_{l_{b}} l_{\beta}(x)[\boldsymbol{\Phi}]^{\mathrm{T}}[\boldsymbol{\Phi}] \mathrm{d} x} \\
& \left\{\boldsymbol{I}_{4}\right\}=\int_{l_{b}} x l_{\alpha}(x)[\boldsymbol{\Phi}]^{\mathrm{T}} \mathrm{d} x=\int_{l_{b}} x l_{\beta}(x)[\boldsymbol{\Phi}]^{\mathrm{T}} \mathrm{d} x \\
& I_{5}=C_{N \alpha} q S=\int_{l_{b}} l_{\alpha}(x) \mathrm{d} x=\int_{l_{b}} l_{\beta}(x) \mathrm{d} x \\
& {\left[\boldsymbol{I}_{6}\right]=\int_{l_{b}} l_{\alpha}(x)\left[\boldsymbol{\Phi}^{\prime}\right] \mathrm{d} x=\int_{l_{b}} l_{\beta}(x)\left[\boldsymbol{\Phi}^{\prime}\right] \mathrm{d} x} \\
& {\left[\boldsymbol{I}_{7}\right]=\int_{l_{b}} l_{\alpha}(x)[\boldsymbol{\Phi}] \mathrm{d} x=\int_{l_{b}} l_{\beta}(x)[\boldsymbol{\Phi}] \mathrm{d} x=\left\{\boldsymbol{I}_{1}\right\}^{\mathrm{T}}} \\
& I_{8}=C_{M \alpha} q S=\int_{l_{b}} x l_{\alpha}(x) \mathrm{d} x=\int_{l_{b}} x l_{\beta}(x) \mathrm{d} x \\
& {\left[\boldsymbol{I}_{9}\right]=\int_{l_{b}} x l_{\alpha}(x)\left[\boldsymbol{\Phi}^{\prime}\right] \mathrm{d} x=\int_{l_{b}} x l_{\beta}(x)\left[\boldsymbol{\Phi}^{\prime}\right] \mathrm{d} x} \\
& {\left[\boldsymbol{I}_{10}\right]=\int_{l_{b}} x l_{\alpha}(x)[\boldsymbol{\Phi}] \mathrm{d} x=\int_{l_{b}} x l_{\beta}(x)[\boldsymbol{\Phi}] \mathrm{d} x=\left\{\boldsymbol{I}_{4}\right\}^{\mathrm{T}}} \\
& I_{11}=\int_{l_{b}} x^{2} l_{\alpha}(x) \mathrm{d} x=\int_{l_{b}} x^{2} l_{\beta}(x) \mathrm{d} x
\end{aligned}
$$




\section{Acknowledgment}

The grant support from National Science Foundation of China, No.10972033, is greatly acknowledged.

\section{References}

[1] Platus DH. Aeroelastic stability of slender, spinning missiles. Journal of Guidance, Control, and Dynamics.1992; 15: 144151.

[2] Mikhail AG. In-flight rod flexure and spin lock-in for kinetic energy projectiles. 20th Atmospheric Flight Mechanics Conference, AIAA-95-3429-CP, 1995.

[3] Mikhail AG. In-flight flexure and spin lock-in for antitank kinetic energy projectiles. Journal of Spacecraft and Rockets. 1996; 33: 657-664.

[4] Murphy $\mathrm{CH}$, Mermagen WH. Flight mechanics of an elastic symmetric missile. Journal of Guidance, Control, and Dynamics. 2001; 24: 1125-1132.

[5] Murphy $\mathrm{CH}$, Mermagen WH. Flight motion of a continuously elastic finned missile. Journal of Guidance, Control, and Dynamics. 2003; 26: 89-98.

[6] Murphy CH, Mermagen WH. Spin-yaw lockin of an elastic finned projectile. Journal of Guidance, Control, and Dynamics. 2005; $28: 121-130$.

[7] Jegarkandi MF, Nobari AS, Sabzehparvar M, Haddadpour H. Aeroelastic stability consideration of supersonic flight vehicle using nonlinear aerodynamic response surfaces. Journal of Fluids and Structures, 2009, 25(6): 1079-1101.

[8] Kitson RC, Cesnik CE. Aeroelastic modeling and simulation of high-speed flexible vehicles. 15th Dynamics Specialists Conference. 2016: 1324.

[9] Shi Z, Zhao L, Peng J. Coning motion instability of a spinning missile induced by aeroelasticity. Atmospheric flight mechanics conference and exhibit. 2016.

[10] Beal TR. Dynamic stability of flexible missile under constant and pulsating thrusts. AIAA Journal. 1965; 3: $486-494$.

[11] Beal TR. Dynamic stability of a uniform free-free beam under a gimbaled thrust of periodically varying magnitude. Stanford University, 1963.

[12] Bolotin VV. Nonconservative problems of the theory of elastic stability. Oxford, UK: Pergamon Press, 1963.

[13] Wu JJ. Missile stability using finite elements - An unconstrained variational approach. AIAA Journal.1976; 14: 313-319.

[14] Park YP. Dynamic stability of a free Timoshenko beam under a controlled follower force. Journal of Sound and Vibration. 1987; 113: 407-415.

[15] Kim JH, Choo YS. Dynamic stability of a free-free Timoshenko beam subjected to a pulsating follower force. Journal of Sound and Vibration. 1998; 216: 623-636. 
[16] Pourtakdoust SH, Assadian N. Investigation of thrust effect on the vibrational characteristics of flexible guided missiles. Journal of Sound and Vibration. 2004; 272: 287-299.

[17] Xu Y, Xie C, Yang C. Effect of thrust on aeroelastic stability of a slender missile. AIAA Conference, Schaumburg, IL, AIAA 2008-2022, 2008.

[18] Wu L, Xie C, Yang C. Aeroelastic stability of a slender missile with constant thrust. Procedia Engineering, 2012, 31: 128135.

[19] Trikha M, Mahapatra DR, Gopalakrishnan S, Pandiyan R. Structural stability of slender aerospace vehicles: Part I Mathematical modeling. International Journal of Mechanical Sciences. 2010; 52: 937-951.

[20] Trikha M, Mahapatra DR, Gopalakrishnan S, Pandiyan R. Structural stability of slender aerospace vehicles: Part II Numerical simulations. International Journal of Mechanical Sciences. 2010; 52: 1145-1157.

[21] Hodges DH. A new approach to aeroelastic response, stability and loads of missiles and projectiles. Final Report, U.S. Army Research Office Grant 40448-EG, 2004.

[22] Rasti A, Fazelzadeh SA. Multibody dynamic modeling and flutter analysis of a flexible slender vehicle[J]. International Journal of Structural Stability and Dynamics, 2012, 12(06): 1250049.

[23] Oliveira EJ, Gasbarri P, Milagre FI. Flight Dynamics Numerical Computation of a Sounding Rocket Including Elastic Deformation Model. AIAA Atmospheric Flight Mechanics Conference. 2015: 2710.

[24] Livshits DS, Yaniv S, Karpel M. Dynamic stability of free flight rockets. Proceedings of the 37th Structures, Structural Dynamics and Materials Conference, Salt Lake City, Utah, 1996: 230-236.

[25] Yoon S-J, Kim j-H. Effect of a concentrated mass on the spinning free-free beam subjected to a thrust. AIAA/ASME/ASCE/AHS/ASC Structures, Structural Dynamics, and Materials Conference and Exhibit, 42 nd, Seattle, WA, 2001.

[26] Huang X, Zeiler TA. Dynamics of flexible launch vehicles with variable mass. 44th AIAA Aerospace Sciences Meeting and Exhibit, Reno, Nevada, AIAA 2006-826, 2006.

[27] Choi SH, Pierre C, Ulsoy AG. Consistent modeling of rotating Timoshenko shafts subject to axial loads. Journal of Vibration and Acoustics. 1992; 114: 249-259.

[28] Wu J, Lei J. Aerodynamic configuration and characteristics of guided weapons. Beijing: National Defense Industry Press, 2008.

[29] Baruh H. Analytical dynamics. New York: McGraw-Hill, 1999.

[30] Meirovitch L, Nelson HD. On the high-spin motion of a satellite containing elastic parts. Journal of Spacecraft and Rockets. 1966; 3: 1597-1602. 
[31] Huang X, Fan H, Jiang H. Aeroelastic analysis of slender Spinning vehicle. Journal of Beijing Institute of Technology. 1998; 18: 431-435. 